\title{
Bubble plumes in a stratified environment: Source parameters, scaling, intrusion height, and neutral height
}

\author{
Shigan $\mathrm{Chu}^{1, *}$ and Andrea Prosperetti ${ }^{2, \dagger}$ \\ ${ }^{1}$ Department of Mechanical Engineering, Johns Hopkins University, Baltimore, Maryland 21218, USA \\ ${ }^{2}$ Department of Mechanical Engineering, University of Houston, Houston, Texas 77204, USA
}

(Received 10 May 2017; published 27 October 2017)

\begin{abstract}
A cross-sectionally averaged model is used to study several aspects of the physics of a bubble plume rising in a stratified quiescent liquid. Scaling analyses for the peel height, at which the plume momentum vanishes, and the neutral height, at which its average density equals the ambient density, are presented. Contrary to a widespread practice in the literature, it is argued that the neutral height cannot be identified with the experimentally reported intrusion height. Recognizing this difference provides an explanation of the reason why the intrusion height is found so frequently to be much above predictions and brings the theoretical results in line with observations. The mathematical model depends on three dimensionless parameters, some of which are related to the inlet conditions at the plume source. Their influence on the peel and neutral heights is illustrated by means of physical considerations, scaling analyses, and numerical results.
\end{abstract}

DOI: 10.1103/PhysRevFluids.2.104503

\section{INTRODUCTION}

Bubble plumes occur in a number of situations such as the aeration or destratification and mixing of water bodies (see., e.g., Refs. [1-4]), submarine volcanic eruptions (see, e.g., Ref. [5]), metal processing (see, e.g., Ref. [6]), and others. A recent occurrence is the major oil spill caused by the Deepwater Horizon accident in the Gulf of Mexico in 2010 [7-9].

The behavior of these plumes is strongly influenced by the degree of stratification of the ambient liquid. In the absence of stratification, the plume reaches fully developed conditions as found, e.g., in Refs. [10-13]. The situation is markedly different if the ambient liquid is stably stratified, as then, with sufficient depth, the plume rises to a maximum height, the peel height. Because of the accumulated momentum in the plume, this height is greater than the level of neutral buoyancy, often referred to as the intrusion, or trap, height. The plume liquid falls back down from the peel height to the intrusion height, giving rise to a complex structure that has stimulated the development of so-called double-plume models $[14,15]$, which have also proved useful in the study of fountains (see. e.g., Refs. $[16,17])$. If the water is deep enough, the pattern can repeat with the appearance of more than a single pair of peel and intrusion heights (see, e.g., Refs. [18,19]).

Other important features determining the plume dynamics are the depth and the horizontal extent of the ambient liquid. Limitations on both quantities permit the coupling of surface waves with the plume buoyant motion, sometimes resulting in sloshing of the liquid surface and meandering of the plume (see. e.g., Refs. [20-24]). If the depth is comparable with the hydrostatic head $p_{\text {atm }} /\left(\rho_{w} g\right.$ ) (with $p_{\text {atm }}$ the atmospheric pressure, $\rho_{w}$ the liquid density, and $g$ the acceleration of gravity), the expansion of the bubbles as they rise and respond to the decreasing hydrostatic pressure impacts the plume behavior (see, e.g., Refs. [14,18]). With stratification, this effect is smaller for water depths shallower than $p_{\text {atm }} /\left(\rho_{w} g\right)$ or, for deeper water bodies with sufficient stratification, up to the first peel height.

The effect of bubble size has been considered by several investigators, including Refs. $[4,18,19,25]$, all of whom focused on the size-dependent slip velocity of the bubbles relative to

\footnotetext{
*schu13@jhu.edu

${ }^{\dagger}$ Corresponding author: aprosperetti@uh.edu; also, Faculty of Science and Technology, Impact Institute and J. M. Burgers Center for Fluid Dynamics, University of Twente, 7500AE Enschede, The Netherlands.
} 
the surrounding liquid. Of course, the assumption of equal-size bubbles is a simplification. Several investigators have developed models to predict the bubble size distribution in an underwater blowout (recent references are Refs. [26,27]), but these models do not seem to have yet been incorporated into full plume models.

Most of the theoretical work on the dynamics of bubble plumes is based on an integral formulation of the balance equations (see, e.g., Refs. $[4,10,12,14,18,25,28]$ ) following the classic approach used for single-phase plumes (see, e.g., Refs. [29-31]). An important result achieved in this work is the identification of the dimensionless quantities having the greatest influence on the plume behavior. More recently, two-fluid models or Lagrangian-Eulerian models based on Navier-Stokes equations with large-eddy turbulence models have been developed (see, e.g., Refs. [32-35]). Reference [36] compared these more recent models with the integral approach and found a good agreement on several fundamental aspects of the plume behavior.

Difficulties are encountered in the attempt to compare the theoretical predictions with the experimentally reported intrusion heights. In the first place, the experimental results appear to be inconsistent with each other and even to violate upper bounds set by the theory (see, for example, Fig. 10 below). Second, the predictions are found to fall significantly below the data. It will be argued that this situation, which was the original motivation for this study, is a consequence of the improper identification of the neutral height, at which the mean plume density equals the ambient density, with the observed intrusion height. Recognizing the difference between these two quantities suggests an explanation for the difficulties just mentioned and reconciles the theoretical results with data from two experiments which report sufficient information to distinguish between the two heights.

A second motivation for this study is a question faced by researchers interested in integral formulations of buoyant plumes, namely the modeling of inlet conditions at the plume source. In the case of single-phase plumes, and bubble plumes in an unstratified environment, the inlet conditions have a negligible influence on the ultimate behavior of the plume (see. e.g., Refs. [10,37]). As recognized in Ref. [38], this conclusion does not hold in a stratified environment where, however, the concept of virtual source proves useful in the case of single-phase plumes. (The virtual source is defined as a pointlike source of momentum, but not of mass, located below the actual source at such a depth that the plume mass and momentum flow rates acquire the correct source values at the depth of the actual source.) Researchers on bubble plumes in stratified environments have dealt with the problem of inlet conditions by using educated guesses based on approximating the starting flow as similar to that in a single-phase plume (see, e.g., Refs. $[4,39,40]$ ). Here we present an attempt at a more general modeling of the inlet conditions, such as momentum and mass flow rates, in the context of a horizontally integrated plume model.

\section{MATHEMATICAL MODEL}

Since our objective here is to focus on the basic physics of bubble plumes, we use a standard, horizontally integrated, quasi-one-dimensional model for an axisymmetric, vertically-oriented plume. The flow is driven by the inlet conditions at the plume source and by the rising motion of the bubbles, which are assumed to have the same size; the bubble size enters the model indirectly through the rise velocity $w_{b}$. The liquid is assumed to be stably vertically stratified with a density $\rho_{a}(z)(z$ is the vertical coordinate positive upward measured from the plume actual source), and there is no cross flow. A similar model has been used by many previous authors (see, e.g., Refs. [4,14,29,30,38,39,41]). Some comments on the derivation of the model equations are provided in the appendix; here we simply state them.

The statement of conservation of the liquid volume is

$$
\frac{d m}{d z}=2 \pi b(z) \alpha_{e} w(z)
$$


with

$$
m(z)=\int_{A}\left(1-\alpha_{b}\right) w d A \simeq \int_{A} w d A,
$$

where $b(z)$ is the local radius of the plume, $w(z)$ is the vertical liquid velocity, and $m$ is the liquid volume flow rate in the plume. In the last step of (2) we have neglected the bubble volume fraction $\alpha_{b}$, which is usually small. In the derivation of (2) from the principle of conservation of mass the liquid density appears both inside the integral and in the right-hand side. We have canceled it, invoking a Boussinesq-type approximation based on the assumption that the difference between the liquid density inside and outside the plume has negligible effects on the mass balance.

In the right-hand side of (1), we have made the usual entrainment hypothesis, valid for fully developed turbulent conditions, which establishes a proportionality relation between the entrainment velocity of the ambient fluid and the plume vertical velocity, the proportionality constant being the entrainment coefficient $\alpha_{e}$. This proportionality relation can be justified on the basis of dimensional considerations noting the irrelevance of viscosity at high Reynolds numbers in fully developed turbulent conditions; similar arguments can be found, e.g., in Secs. 35 and 36 of Ref. [42] or Chap. 5 of Ref. [43]. On the same basis, provided fully developed conditions prevail, the numerical value of $\alpha_{e}$ can be expected to be independent of the physical properties of the liquid. (Because of this hypothesis, the considerations that follow are not directly applicable to laminar bubbly plumes.) In the literature on integral models of bubble plumes with a Gaussian distribution of the flow fields (density, velocity etc.), frequently used values are $\alpha_{e}=0.083-0.085$ [18,41,44]. Experimental values encountered in the literature are between 0.07 and 0.11 [45]. This range is supported by the recent direct numerical simulations (DNS) of single-phase plumes in Ref. [46], where, however, a dependence on distance from the source was found. The recent large-eddy numerical simulations (LES) of bubble plumes in Ref. [35] also find that $\alpha_{e}$ depends on distance from the source. The mean value over the entire height of the simulation reported by these authors is 0.086 and over the fully developed region is 0.067. A likely explanation for this distance dependence is the existence of a transition zone to fully developed conditions. Since we are interested here in the basic aspects of buoyant bubble plumes rather than precise numerical values, we feel justified in assuming a constant value for $\alpha_{e}$. In the first part of the paper, we do not need to commit ourselves to a specific value because $\alpha_{e}$ will be absorbed in dimensionless groups. When we come to a comparison with experiment, we find that the accuracy of the data does not permit too fine a discrimination among the various values of $\alpha_{e}$ reported in the literature.

The results of Ref. [35] have a bearing also on the entrainment process in the case of "pseudoturbulent" plumes, namely plumes in which the disordered liquid motion is driven primarily by the rising bubbles themselves, because the influence of the velocity imparted by the source will have dissipated in the far field. In this region, with their Gaussian model, the authors find an average value $\alpha_{e} \simeq 0.067$, which is equivalent to $0.067 \sqrt{2}=0.095$ for the top-hat profile used in this paper. Aside from the precise value, the important point is that modeling entrainment with a constant coefficient seems applicable to a pseudoturbulent plume as well. This conclusion appears plausible again on purely dimensional grounds since experiment shows that the bubble size and their mean distance are irrelevant [47]. The feature introduced by the bubbles is a spatial scale of the order of $w_{b}^{2} / g$, with $w_{b}$ being the rise velocity of the bubbles. For a typical value $w_{b} \sim 300 \mathrm{~mm} / \mathrm{s}$, we have $w_{b}^{2} / g \sim 9 \mathrm{~mm}$. The scale of the turbulent eddies responsible for entrainment is the integral scale, of the order of the plume diameter $b$, which is much larger. For this reason, the scale $w_{b}^{2} / g$ cannot significantly affect the entrainment is process and therefore should not be included in dimensional considerations.

With the neglect of diffusion at the plume edge, the balance equation for the dissolved salt concentration $c$ can be written as

$$
\frac{d}{d z} \int_{A}\left(1-\alpha_{b}\right) \rho w\left(c-c_{a}\right) d A+\frac{d c_{a}}{d z} \int_{A}\left(1-\alpha_{b}\right) \rho w d A=0
$$


in which $c_{a}$ is the concentration in the ambient liquid surrounding the plume. Upon setting

$$
\frac{\rho-\rho_{w}}{\rho_{w}}=\beta_{c} c
$$

where $\rho_{w}$ is the density of pure water and $\beta_{c}$ is a constant, we have

$$
\frac{\rho-\rho_{a}}{\rho_{w}}=\beta_{c}\left(c-c_{a}\right)
$$

and the previous equation may be rewritten as

$$
\frac{d}{d z} \int_{A}\left(1-\alpha_{b}\right) \frac{\rho-\rho_{a}}{\rho_{w}} g \rho w d A=N^{2} \rho_{w} \int_{A}\left(1-\alpha_{b}\right) \rho w d A,
$$

with

$$
N^{2}=-\frac{g}{\rho_{w}} \frac{\partial \rho_{a}}{\partial z}
$$

the Brunt-Väisäla frequency. As before, we invoke the Boussinesq approximation and the smallness of $\alpha_{b}$ to rewrite this equation in the approximate form

$$
\frac{d F_{s}}{d z}=-N^{2} m
$$

where the buoyancy flux due to salinity, $F_{s}$, is defined by

$$
F_{s}=\int_{A} \frac{\rho-\rho_{a}}{\rho_{w}} g w d A .
$$

Although here and in the following we explicitly refer to a density stratification due to salinity, as far as the liquid is concerned, these expressions are also applicable to the case of thermal stratification with an obvious reinterpretation of the symbols. Thermal effects, however, may affect the bubbles by causing evaporation and condensation. These processes would have limited effects if the partial pressure of the vapor remains small compared to that of the gas and if the bubble volume fraction is small so that latent heat effects have a negligible impact on the liquid temperature.

The buoyancy flux due to the bubbles is defined similarly to (9) as

$$
F_{b}=\int_{A} \frac{\rho-\rho_{g}}{\rho_{w}} g\left(w+w_{b}\right) \alpha_{b} d A \simeq g \int_{A}\left(w+w_{b}\right) \alpha_{b} d A=g Q_{b},
$$

in which $\rho_{g} \ll \rho$ is the gas density and $Q_{b}$ is the gas flow rate injected at the plume source. In the present paper, we assume that the change in the bubble volume is negligible, an assumption that is justified for plumes in shallow water or for a fraction of the plume development in deep water as noted before. With this approximation, $Q_{b}$ remains constant so that the counterpart of (8) for the bubble buoyancy flux is simply

$$
\frac{d F_{b}}{d z}=0
$$

The bubbles exert a drag force on the liquid which, since the acceleration to which they are subjected is very small, essentially balances the buoyancy force acting on them. Thus, the force exerted by the bubbles on the liquid can be replaced by their buoyancy in the liquid momentum equation. The liquid momentum equation then becomes (see the Appendix)

$$
\frac{d M}{d z}=B_{b}+B_{s}
$$


where

$$
\begin{gathered}
M=\int_{A}\left(1-\alpha_{b}\right) w^{2} d A \simeq \int_{A} w^{2} d A, \\
B_{b}=\int_{A} \alpha_{b} \frac{2 \rho-\rho_{a}-\rho_{g}}{\rho_{w}} g d A \simeq \int_{A} \alpha_{b} g d A, \\
B_{s}=\int_{A} \frac{\rho_{a}-\rho}{\rho_{w}} g d A .
\end{gathered}
$$

Here $M$ can be identified with the momentum flow rate per unit mass of the liquid, $B_{b}$ with the buoyancy due to bubbles and $B_{s}$ with the buoyancy due to salinity (or temperature). In defining the momentum flow rate $M$, we have assumed a unit value for the so-called momentum amplification factor which accounts in an approximate way for the presence of turbulence [12]. The commonly used value for this parameter is about 1.1 (see, e.g., Refs. [12,15]). Its inclusion would introduce another empirical parameter without altering significantly our results.

The two terms in the plume momentum equation (12) have opposite signs because the rising plume liquid is saltier (or colder) and therefore heavier than the surrounding liquid. A level therefore is reached where $d M / d z=0$; we term this the neutral height. Above this level, the plume gets progressively heavier than the surrounding fluid, starts to slow down, and after reaching the peel height where $M=0$, falls back, settling at what is referred to as the intrusion height. Representing this final stage in an integral model requires the adoption of a double-plume model such as that developed, e.g., in Refs. [14,15]. In the framework of the present model, one simply observes that, as the plume rises toward the peel height, it slows down and its cross section increases to conserve mass until it diverges at the peel height, where its momentum vanishes. This divergence is an artifact of the present and other single-plume models, which, however, does not affect our main conclusions, as will be clear from the developments that follow.

As pointed out in Refs. [30,44], the neutral height, where $d M / d z=0$, represents an estimate of the lower edge of the intrusion layer, which experimentally is identified by observing the level at which the liquid falling back from the peel height expands horizontally. The reason why this is a lower bound is that this falling liquid is lighter than the plume liquid at the neutral level, due to the lighter liquid entrained on the way up to the peel height. This observation has important implications for the comparison of theory and experiment, as will be shown below in Sec. VI.

In summary, in integral form the equations of the model are given by (1), (8), (11), and (12). Further progress requires an assumption on the distribution of the fields over the plume cross section. Following approximations common in the literature, we assume either a top-hat or a Gaussian form. For the top-hat model (see, e.g., Ref. [38]), we have

$$
m=\pi w b^{2}, \quad M=\pi w^{2} b^{2}, \quad F_{s}=\pi w \frac{\rho-\rho_{a}}{\rho_{w}} g b^{2} .
$$

After substitution of (16) into the governing equations (1), (8), and (12), we find

$$
\begin{gathered}
\frac{d m}{d z}=2 \sqrt{\pi} \alpha_{e} \sqrt{M}, \\
\frac{d M}{d z}=\frac{Q_{b} g}{w+w_{b}}+\frac{F_{s}}{w}, \\
\frac{d F_{s}}{d z}=-m N^{2} .
\end{gathered}
$$

We have assumed that the gas and salinity distributions are uniform over the entire plume cross section rather than localized in inner regions; equations for the more general case are given in the 
appendix for the Gaussian profile. There it is also shown that a suitable redefinition of the scaled variables puts these more general equations in the same dimensionless form used below, on which our scaling results are based.

As already mentioned, one of the important points of this paper is the focus on the neural height, defined as the height above the source where the plume density equals the ambient density. These conditions correspond to the maximum value of the momentum flux and are found by setting to zero the right-hand side of (18):

$$
F_{s}=-\frac{w}{w+w_{b}} Q_{b} g
$$

The mechanism by which this relation gives the neutral height can be seen more clearly by noting that integration of (19) gives a relation $F_{s}=F_{s}(z)$. Since $F_{s}$ is monotonically decreasing with $z$, this relation can be inverted to give $z=z\left(F_{s}\right)$. The neutral height is then found by substituting in this relation the right-hand side of (20) in place of $F_{s}$. It is therefore evident that the neutral height will be the lower the faster $m$ increases. In particular, then, the neutral height will be a monotonically decreasing function of the momentum injected by the source which increases $d m / d z$ as shown by Eq. (17).

\section{SINGLE-PHASE PLUME}

For purposes of orientation, it is useful to consider the single-phase plume first. Furthermore, when the bubble rise velocity is very small, the bubbly mixture behaves essentially like a homogeneous lighter liquid and the single-phase results are directly applicable.

Two important limit cases are those of a plume dominated by the salinity buoyancy flux $F_{s 0}$ or one dominated by the momentum $M_{0}$ injected by the source. In the former case, the momentum equation (12) is unimportant and the order of magnitude of the height $L_{b}$ reached by the plume in this buoyancy-dominated case is dependent on the mass (17) and buoyancy (19) equations. The characteristic time scale is $N^{-1}$ so that $w \sim L_{b} N$ and, from the two equations, we have the estimates

$$
\frac{m_{0}}{L_{b}} \sim 2 \pi \alpha_{e} b L_{b} N, \quad-\frac{F_{s 0}}{L_{b}} \sim-m_{0} N^{2} .
$$

Here and in the rest of the paper, the index 0 denotes values at the source. Upon using (16) in the mass conservation equation (17), we have the estimate

$$
\frac{\pi b^{2} w}{z} \sim 2 \pi \alpha_{e} w b
$$

which implies a linear dependence of the plume radius upon the height above the source, $b \sim 2 \alpha_{e} z$, in particular, just below the peel height $L_{b}, b \sim 2 \alpha_{e} L_{b}$. Upon using this relation and eliminating $m_{0}$ between the two relations (21), we find

$$
L_{b}=\left(\frac{F_{s 0}}{4 \pi \alpha_{e}^{2} N^{3}}\right)^{1 / 4},
$$

where the equal sign defines the quantity in the left-hand side.

In the momentum-dominated case, it is the buoyancy equation that plays a minor role. If, on the basis of (9) and (15), we use the estimate $F_{s} \sim w B_{s} \sim L_{m} N B_{s}$, we obtain from the momentum equation (12)

$$
-\frac{M_{0}}{L_{m}} \sim \frac{F_{s}}{L_{m} N}
$$

where the minus sign in the left-hand side arises from the fact that $M \simeq 0$ for $z \simeq L_{m}$. Upon substituting the estimate of $F_{S}$ found from this equation into (23), we find

$$
L_{m}=\left(\frac{M_{0}}{4 \pi \alpha_{e}^{2} N^{2}}\right)^{1 / 4} \text {. }
$$


These results agree with those given in Ref. [30]. A derivation of the analogous result for the bubble plume case will be given below in Sec. V. The ratio $\left(L_{m} / L_{b}\right)^{8}$ is the parameter $S$ previously introduced in the literature (see, e.g., Ref. [50]):

$$
S=\left(\frac{L_{m}}{L_{b}}\right)^{8}=\left(\frac{M_{0} N}{F_{s 0}}\right)^{2} .
$$

Upon using (16) we see that $M_{0} N / F_{s 0} \propto \rho_{w} w_{0} N /\left[\left(\rho-\rho_{a}\right) g\right]$ so that $\sqrt{S}$ can be seen as the ratio of the mean rate of change of the plume momentum to the reduced gravity force. Large and small values of $S$ correspond therefore to strong and weak accelerations of the plume liquid, respectively.

The Froude number, defined by

$$
\operatorname{Fr}=\frac{w}{\sqrt{2 \lambda_{s} b g\left(\rho_{a}-\rho\right) / \rho}}
$$

is often used to represent the ratio of inertia and buoyancy of the plume (see e.g., Ref. [4]). Here $\lambda_{s}$ denotes the fraction of the plume radius where the salinity differs from the ambient value. In a uniform ambient, Fr reaches an asymptotic value as the plume becomes fully developed (see, e.g., Ref. [30]). For a top-hat profile, this asymptotic value can be deduced from the results of Ref. [30] and is

$$
\operatorname{Fr}_{\infty}=\sqrt{\frac{5 \lambda_{s}}{16 \alpha_{e}}}
$$

The result for a Gaussian profile is recovered by substituting $\alpha_{e}$ by $\alpha_{e} / \sqrt{2}$, as can be seen upon comparing (17) and (A29). If the Froude number at the source is larger than this asymptotic value, the plume is termed "forced," while if it is smaller, it is termed "lazy" [41]. The inverse square of the ratio of the source Froude number to the asymptotic value

$$
\Gamma=\frac{5 \lambda_{s}^{2}}{8 \alpha_{e}} \frac{b_{0} g\left(\rho_{a 0}-\rho_{0}\right) / \rho_{0}}{w_{0}^{2}}
$$

can then be used to characterize the strength of the source. By using (16) we may re-express this quantity as

$$
\Gamma=\frac{5}{8 \sqrt{\pi} \alpha_{e}} \frac{F_{s 0} m_{0}^{2}}{M_{0}^{5 / 2}} .
$$

Forced plumes correspond to $\Gamma<1$ and lazy ones to $\Gamma>1$. An alternative way to regard the dimensionless parameter $\Gamma$ is as the ratio of two length scales, one,

$$
L_{Q} \sim \frac{b_{0}}{\alpha_{e}} \sim \frac{m_{0}}{\alpha_{e} \sqrt{M_{0}}}
$$

measuring the distance of the source from the virtual source of the plume (where its radius and mass flux vanish), and the other, $L_{M}$, characterizing the height above the source over which the momentum flux dominates. This latter scale can be estimated by noting the relation $m \sim \alpha_{e} L_{M} \sqrt{M}$, from (17), and substituting into (18) written as $M_{0} / L_{M} \sim F_{s 0} m_{0} / M_{0}$ to find

$$
L_{M} \sim\left(\frac{M_{0}^{3 / 2}}{\alpha_{e} F_{s 0}}\right)^{1 / 2} \sim \frac{b_{0}}{\alpha_{e} F r} .
$$

With these estimates, (30) follows upon forming the ratio $\left(L_{Q} / L_{M}\right)^{2}$ [41]. In this picture, $\Gamma>1$ means that momentum effects have exhausted themselves before the actual source height is reached, i.e., that the plume is buoyancy dominated. 
While $S$ and $\Gamma$ both contain buoyancy and momentum, the key difference between the two is the presence of the characteristic time $N$ in the former. As a consequence, $S$ compares the rate of change of the plume velocity with the gravitational acceleration, rather than the plume velocity with the characteristic fall velocity (or the plume kinetic energy with the gravitational potential energy) as $\Gamma$ does. Thus, $S$ embodies the effect of forces, while $\Gamma$ compares energies. This parameter does not include turbulence which, as mentioned above, would increase the plume momentum flux by about $10 \%$ and, therefore, the plume kinetic energy by a comparable amount. This is not completely negligible, but inclusion of turbulent energy will not change the orders of magnitude, which are our focus here.

The mathematical model described in the previous section can be solved analytically for a single-phase plume. The solution is somewhat involved and is given in the appendix. Here we simply quote results for the peel height found in the two limit cases considered above for a plume generated by a point source, which are $h_{p} / L_{m}=1.43$ for $S$ large (momentum dominated) and $h_{p} / L_{b}=2.57$ for $S=0$ (buoyancy dominated), respectively. The latter result agrees with that given in Ref. [29] (see also Refs. [48,49]), while the former one can be recovered by accounting for the different normalizations. The results also agree with those of Ref. [50] upon taking $\alpha_{e}=0.12$ in the definitions of $L_{b}$ and $L_{m}$ as in that paper. For the buoyancy-dominated case, the neutral height is found to be $h_{n} / L_{b}=1.95$, while it vanishes for a point source in the momentum-dominated case. The appearance of constants of order 1 in these relations supports the scaling arguments used to deduce (23) and (25).

\section{BUBBLE PLUMES}

In a bubble plume, the total buoyancy flux at the source is the sum of the salinity component $F_{s 0}$ and the bubble component $Q_{b} g$. Unless the gas flow rate is very small, the latter dominates so that source buoyancy flux can be approximated by $Q_{b} g$. With this approximation, the length scale (23) for buoyancy-dominated plumes becomes

$$
L_{b}=\left(\frac{Q_{b} g}{4 \pi \alpha_{e}^{2} N^{3}}\right)^{1 / 4} .
$$

Rather than relying on an analogy, it may be more satisfactory to derive this result starting from the governing equations. On the assumption that the salinity flux is negligible compared with the buoyancy induced by the bubbles, operating as in the previous section, from the momentum equation (18) we find

$$
\frac{\pi b^{2} w^{2}}{L_{b}} \sim \frac{Q_{b} g}{w\left(1+w_{b} / w\right)} .
$$

Upon setting $w \sim N L_{b}$ and $b \sim 2 \alpha_{e} L_{b}$, we then have

$$
L_{b}=\left[\frac{Q_{b} g}{4 \pi \alpha_{e}^{2} N^{3}\left(1+w_{b} / N L_{b}\right)}\right]^{1 / 4} .
$$

This result makes evident that the height reached by the plume is a decreasing function of the bubble-liquid slip velocity $w_{b}$, as will be confirmed in the numerical examples of the next section. Physically, this happens because large values of the bubble rise velocity imply that the bubbles are unable to exert a strong drag force on the liquid and lift it. If the new dimensionless parameter $w_{b} /\left(L_{b} N\right)$ appearing in (35) is very small, the result is the same as (33) and justifies the previous derivation. If, on the other hand, $w_{b} / L_{b} N \gg 1$, (35) leads to the new scale

$$
L_{b}^{\prime}=\left(\frac{Q_{b} g}{4 \pi \alpha_{e}^{2} N^{2} w_{b}}\right)^{1 / 3} \text {. }
$$


Numerically, it is found that the peel and neutral heights nondimensionalized by this length scale are approximately independent of the parameters of the problem for $V_{N}$ larger than about 5. A convenient nondimensionalization of $w_{b}$ is through the definition of the parameter $[14,19]$

$$
V_{N}=w_{b}\left(\frac{4 \pi \alpha_{e}^{2}}{N Q_{b} g}\right)^{1 / 4},
$$

which equals $w_{b} /\left(L_{b} N\right)$ if $L_{b}$ is taken from (33).

To adapt the parameter $\Gamma$ characterizing the source strength to the bubble plume case, we replace $F_{s 0}$ by $Q_{b} g$ in the definition (30). According to Ref. [10], the asymptotic value of the Froude number for a bubble plume in a uniform ambient liquid is given by $\sqrt{3 \lambda_{b} / 8 \alpha_{e}}$, with $\lambda_{b}$ the fraction of the plume radius occupied by the bubbles. However, in the literature, the normalization most frequently used is the same as for the single-phase case (see, e.g., Refs. [13-15] and, accordingly, we define $\Gamma$ as

$$
\Gamma=\frac{5}{8 \alpha_{e}} \frac{Q_{b} g m_{0}^{2}}{\sqrt{\pi} M_{0}^{5 / 2}} .
$$

By using (16) for a top-hat profile, we see that $\Gamma \propto\left(Q_{b} g /\left[w_{0}\left(\alpha_{e} w_{0}^{2} b_{0}\right)\right]\right.$, which may be seen as proportional to the ratio of the buoyancy force acting on the gas, of the order of $\rho\left(Q_{g} / N\right) g$, to the rate at which the momentum of the entrained liquid, of the order of $\rho \alpha_{e} w_{0}^{2} b^{2} / N$, increases as shown by (1).

Finally, we replace $F_{s 0}$ by $Q_{b} g$ in the definition (26) of the parameter $S$, writing

$$
S=\left(\frac{M_{0} N}{Q_{b} g}\right)^{2} .
$$

Rewriting this expression as $\sqrt{S}=\rho M_{0} N /\left(\rho Q_{b} g\right)$, we see that $\sqrt{S}$ represents the rate of change of the plume momentum to the buoyancy force on the gas and has therefore a physical meaning similar to that in the single-phase case. Alternatively, since $Q_{b} / N$ is the total amount of gas injected during the characteristic time $N^{-1}, S$ may also be interpreted as the ratio of the momentum flow rate to the buoyancy force. Buoyancy-dominated plumes, therefore, are characterized by small values of $S$. It may also be noted that

$$
\Gamma \sqrt{S}=\frac{5}{8 \sqrt{\pi} \alpha_{e}} \frac{N m_{0}^{2}}{M_{0}^{3 / 2}}=\frac{5}{8 \sqrt{\pi}} \frac{b_{0} / \alpha_{e}}{w_{0} / N},
$$

with the last step appropriate for a top-hat profile. Recalling that $b \sim \alpha_{e} z$, we see that $\Gamma \sqrt{S}$ may be interpreted as the ratio of the virtual source height to the distance traveled by the plume liquid during the characteristic time $N^{-1}$.

In order to go beyond the scaling analysis and calculate precise numerical values, it is useful to recast the model equations in dimensionless form using the dimensionless independent variable

$$
z^{*}=\frac{z}{L_{b}}
$$

and the definitions

$$
\begin{gathered}
m^{*}=\frac{m}{\pi\left(2 \alpha_{e} L_{b}\right)^{2} N L_{b}}, \quad M^{*}=\frac{M}{\pi\left(2 \alpha_{e} L_{b}\right)^{2}\left(N L_{b}\right)^{2}} \\
B_{b}^{*}=\frac{B_{b}}{\pi\left(2 \alpha_{e} L_{b}\right)^{2} N^{2} L_{b}}, \quad F_{s}^{*}=\frac{F_{s}}{\pi\left(2 \alpha_{e} L_{b}\right)^{2}\left(N L_{b}\right)\left(N^{2} L_{b}\right)} .
\end{gathered}
$$


As mentioned earlier, the combination $2 \alpha_{e} L_{b}$ is of the order of the plume diameter at the height $L_{b}$ above the source. In this way, the equations become

$$
\begin{gathered}
\frac{d m^{*}}{d z^{*}}=\sqrt{M^{*}}, \\
\frac{d M^{*}}{d z^{*}}=B_{b}^{*}+B_{s}^{*}=\frac{1}{M^{*} / m^{*}+V_{N}}+\frac{m^{*} F_{s}^{*}}{M^{*}} \\
\frac{d F_{s}^{*}}{d z^{*}}=-m^{*} .
\end{gathered}
$$

Here we have used the relation $w^{*}=M^{*} / m^{*}$, which is an immediate consequence of (16). When $V_{N}$ is much greater than the liquid velocity $M^{*} / m^{*}$, the weak bubble drag has the consequence that (45) becomes identical to the momentum equation for a single-phase plume.

The corresponding inlet conditions can be expressed in terms of the dimensionless parameters introduced earlier with the results

$$
m_{0}^{*}=\frac{2}{\sqrt{5}} \sqrt{S^{5 / 4} \Gamma}, \quad M_{0}^{*}=S^{1 / 2}
$$

Since, as already noted, the buoyancy flux at the inlet is dominated by the bubbles, we will solve these equations with the initial condition

$$
F_{s 0}^{*}=0 .
$$

The liquid in the rising plume is always heavier than the surrounding liquid, and the buoyancy flux due to salinity vanishes at the source. Thus, $F_{s}^{*}$ is always negative and monotonically increases in magnitude.

As mentioned in the introduction, the inlet conditions used in previous theoretical work adopting the integral model (see, e.g., Refs. [14,39]) are equivalent to taking $S=0$, i.e., a totally buoyancydriven plume with no inlet liquid momentum, and $\Gamma=1$, which is appropriate asymptotically for fully developed plumes in an unstratified environment. This procedure was justified by the results of Refs. [30,40], which suggest that the plume behavior is only weakly dependent on the inlet conditions. While this is approximately true in unstratified environments, we find that the finiteness of the peel and neutral heights in the presence of stratification causes inlet conditions to acquire a greater importance. This result is similar to the case of fountains in which the height reached by the liquid is also finite, not because of stratification but because of the strong effect of gravity [17].

\section{RESULTS}

As formulated, the solution to the mathematical problem embodied in (44), (45), (47), and (48) depends on three parameters, the dimensionless bubble velocity, $V_{N}$, the ratio of the momentum flux to the bubble buoyancy, $S$, and the source strength, $\Gamma$.

The strongest effect of the bubbles is found when no liquid is injected at the source as, in this case, the entire momentum of the plume is provided by the buoyancy of the gas. In this case $S$ $=0$ which, by (47), renders the results independent of $\Gamma$. The solution depends now only on the parameter $V_{N}$, the dimensionless bubble rise velocity. Figure 1 shows the dimensionless peel height $h_{p} / L_{b}$ and the dimensionless neutral height $h_{n} / L_{b}$ for this situation. This result for $h_{n}$ when $S=0$ represents an upper limit for the neutral height as can be deduced from the fact that the height above the source reached by any real plume would be smaller by the distance $L_{Q}$ from the virtual source defined above in (31). The same argument cannot be applied to the peel height as, unlike $h_{n}$ (see the argument at the end of Sec. II) this quantity is not a monotonic function of $S$ as can be seen, for example, in Fig. 5(a) below. When the bubble rise velocity is very small, the bubbles and the liquid form a homogeneous buoyant bubbly mixture. In this case, $w_{b} \ll w$ or $V_{N} \ll M^{*} / m^{*}$ and 


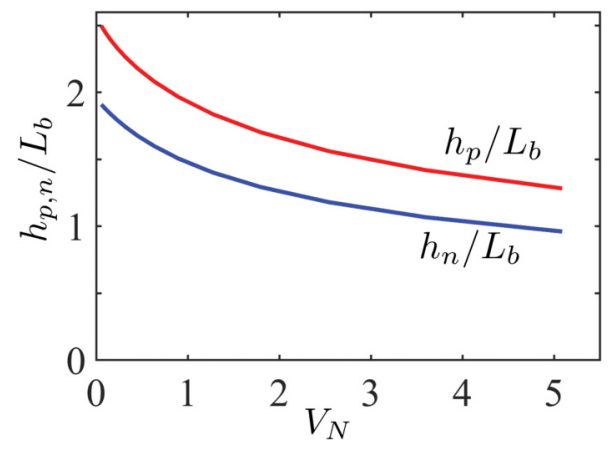

FIG. 1. Normalized peel height $h_{p} / L_{b}$ (upper curve) and neutral height $h_{n} / L_{b}$ for $S=0$ as functions of the dimensionless bubble rise velocity $V_{N}$ defined in (37). The parameter $\Gamma$ has no influence when $S=0$. The neutral height is the level at which the plume momentum attains its maximum value.

the momentum equation (45) reduces to

$$
\frac{d M^{*}}{d z^{*}}=\frac{F_{s}^{*}+1}{w^{*}}
$$

which has the same form as for a single-phase plume wth $F^{*}$ replaced by $F^{*}+1$; the solution is given in the Appendix.

The general trend of the dimensionless peel height $h_{p} / L_{b}$ and neutral height $h_{n} / L_{b}$ shown in Fig. 1 is found even for nonzero values of $S$ and $\Gamma$ when the bubble buoyancy is dominant. This is the case of greatest interest in practice as, in most of the available experiments, little or no liquid is injected at the source, which results in small values of $S$ (see the next section). As expected on the basis of the considerations made in connection with (35), both the peel and neutral heights are decreasing functions of the dimensionless bubble rise velocity $V_{N}$. As the parameter $S$ increases by increasing $M_{0}$ but keeping $Q_{b} g$ fixed (and, therefore, $L_{b}$ fixed as well), a constant value of $\Gamma$ requires the mass discharged by the source to increase [see the definition (38) of $\Gamma$ ]. It is evident from the salinity buoyancy flux equation (19) that a larger $m$ causes $F_{s}$ to become negative faster, which has an adverse effect on the plume momentum as shown by the momentum equation (18). Furthermore, since the source injects heavy fluid, the plume is heavier, the negative buoyancy effect is stronger, and both the peel and neutral heights decrease as shown in Fig. 2.
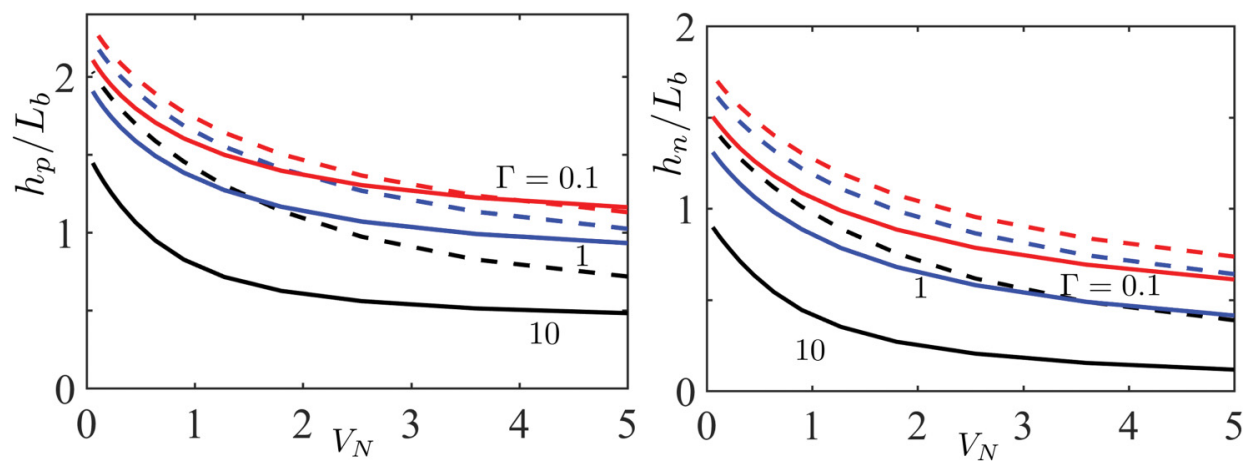

FIG. 2. Typical dependence of the normalized peel height (left) and neutral height on the parameters of the problem. Both the solid lines and dashed lines are, in descending order, for $\Gamma=0.1,1$, and 10; the solid lines are for $S=0.1$; and the dashed lines are for $S=0.01$. 

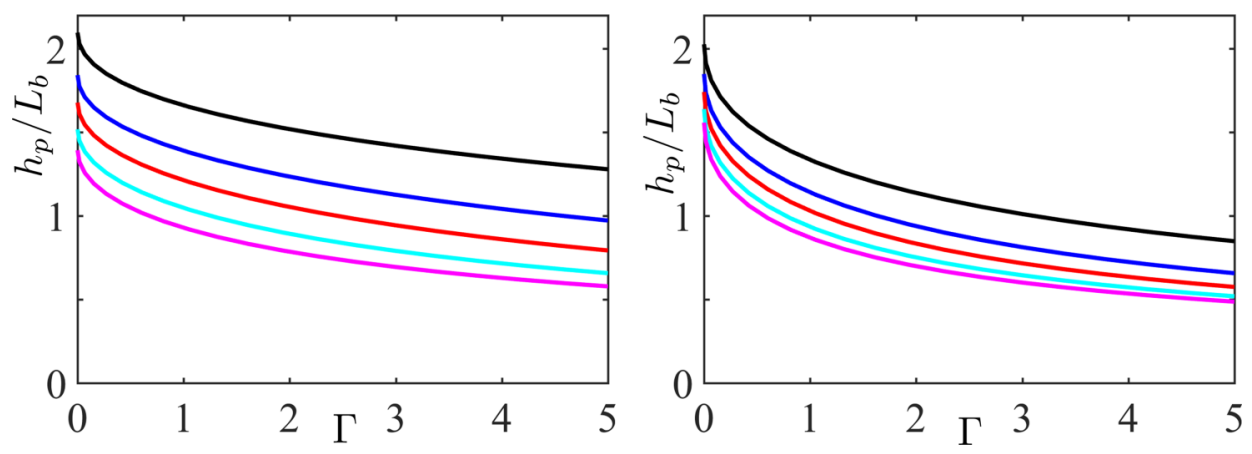

FIG. 3. Normalized peel height for $S=0.25$ (left) and $S=1$ as a function $\Gamma$ for $V_{N}=0.1,0.5,1,2$, and 4 in descending order. Increasing $\Gamma$ makes the plume heavier by increasing the source mass injection and the peel height decreases.

As for the effect of the parameter $\Gamma$ for fixed $S$, the definition (38) shows that an increase in $\Gamma$ implies an increase of the mass discharged by the source so that, again, the peel and neutral heights decrease as shown in Fig. 3. The parameters $\Gamma$ and $S$ can also be varied by varying $Q_{b} g$, which, however, complicates the analysis as $L_{b}$ would then change as well and changes in $h_{p} / L_{b}$ cannot be solely imputed to changes in $h_{p}$.

The decrease of the peel height with $S$ just described prevails, provided the buoyancy imparted by the bubbles is more important than the momentum injected by the source. However, as explained before, by increasing the bubble rise velocity and, with it, $V_{N}$, the effect of the bubble buoyancy decreases so that the momentum injected by the source progressively becomes more important. Thus, while $h_{p} / L_{b}$ decreases with $S$ as in the normal case as long as $V_{N}$ is small and as long as $S$ is small or moderate, it eventually starts increasing for higher $V_{N}$ as shown in Figs. 4 and 5. For large values of $S$, the source momentum prevails also for small $V_{N}$ and $h_{p}$ increases monotonically with $S$ as long as $\Gamma$ (i.e., the mass injected by the source) is small [Figs. 4(a) and 5(a)]. For larger values of $\Gamma$ [Fig. 5(b)], the source mass and the increasing entrainment with $S$ make the plume heavier and $h_{p}$ decreases with $S$. The trend would reverse for $S$ large enough, but large values of this parameter are seldom relevant in practice.

Since the neutral height is determined by the balance of bubble and salinity buoyancy, it is less sensitive to the source momentum and, as $S$ increases, it decreases, maintaining the same qualitative behavior described before and shown in Fig. 2(b), in agreement with the considerations given above at the end of Sec. II.
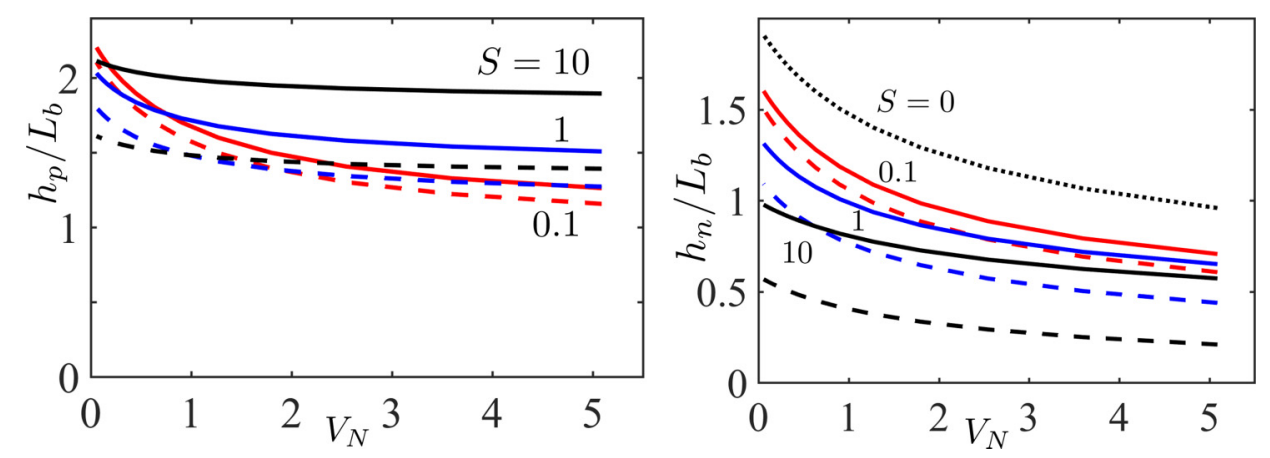

FIG. 4. The left figure shows the normalized peel height for $S=0,0.1,1$, and 10, in decreasing order at the right of the diagram. The right figure is the neutral height with the curves ordered in the opposite direction. The solid and dashed lines correspond to $\Gamma=10^{-3}$ and $\Gamma=10^{-1}$. 

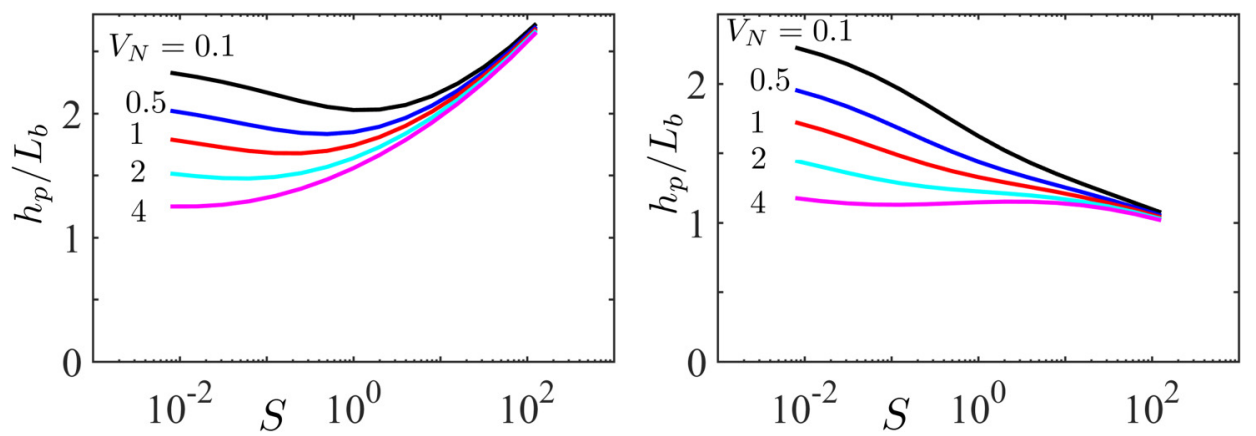

FIG. 5. Normalized peel height vs $S$ for $\Gamma=0$ (left) and $\Gamma=1$; the dimensionless bubble rise velocities are, in descending order, $V_{N}=0.1,0.5,1,2$, and 4 .

The panels in Fig. 6 show the profiles of the momentum flow rate, mass flow rate, plume radius, and salinity buoyancy flux for a fairly large bubble velocity, $V_{N}=2.54$, a small source strength, $\Gamma$ $=0.01$, and several values of $S$. The plume source is located at $z^{*}=0$. Since the total buoyancy (bubbles + salinity) is fairy small, the momentum flux exhibits a very weak growth up to its maximum (i.e., the neutral height) and then decreases to zero at a faster rate as the peel height is approached. The mass flux remains finite, however, as the vanishing velocity is balanced by an increase of the
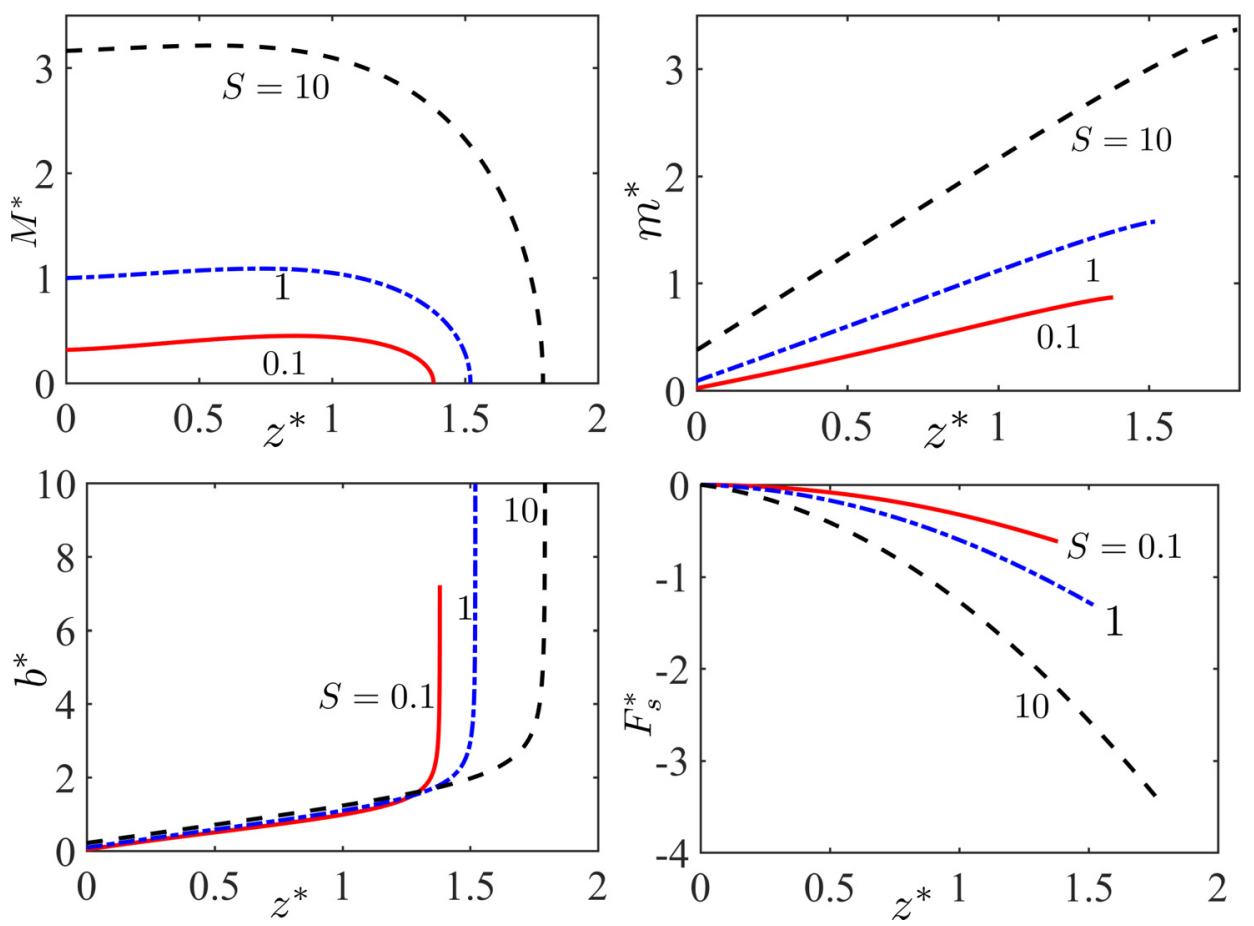

FIG. 6. Vertical distribution of the dimensionless momentum flux $M^{*}$, dimensionless mass flux $m^{*}$, dimensionless plume radius $b^{*}$, and dimensionless salinity buoyancy flux $F_{s}^{*}$ for $V_{N}=2.54, \Gamma=0.01$, and $S=0.1,1$, and 10 . The plume source is at $z^{*}=0$. In these cases, the total buoyancy (bubbles + salinity) is fairy small. Note the divergence of the plume radius as the peel height is approached. The buoyancy flux due to salinity is negative as the plume liquid becomes progressively heavier than the ambient liquid. 

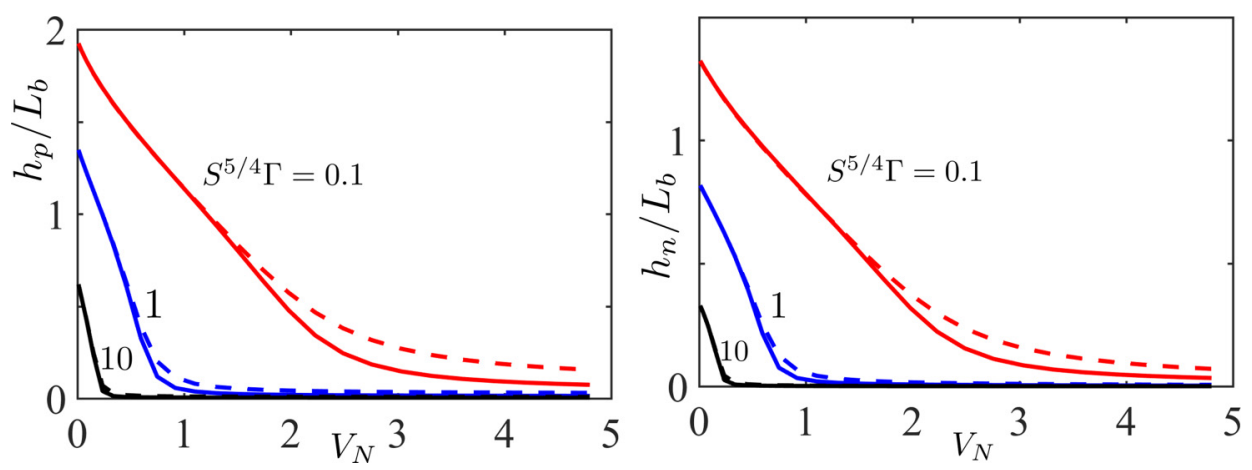

FIG. 7. Normalized peel height (left) and neutral height for a "lazy" plume generated by a source injecting a finite mass of fluid with small momentum. The pairs of curves correspond to $m_{0}^{*} \propto S^{5 / 4} \Gamma=0.1,1$, and 10 . For each pair of curves, the lower one (solid) is for $S=10^{-4}$ and the upper one (dashed) is for $S=5 \times 10^{-4}$.

plume radius at the peel height, where in fact it diverges, as mentioned before and as shown in the third panel. This feature is an artifact of the neglect of the descent of the plume liquid back to the intrusion height, accounting for which would require the adoption of a double-plume model, as explained earlier. For this reason, our result may be considered as an upper limit on the observed peel height. The arguments that follow in Sec. VI focus on the neutral height and are unaffected by this overestimate. The buoyancy flux due to salinity is negative as explained after (48). As the momentum effects compared to buoyancy become larger, i.e., the larger $S$, then $M^{*}, m^{*}, b^{*}$, and $\left|F_{s}^{*}\right|$ also are larger.

For a lazy plume generated by a source injecting a finite mass of fluid with a small momentum, the main agent lifting the liquid is the bubble buoyancy. In this case $S$ is small, but $\Gamma$ is large enough that $m_{0}^{*} \propto \sqrt{\Gamma S^{5 / 4}}$ is finite [see the definition (47) of $m_{0}^{*}$ ]. For small momentum, entrainment in the plume is weak as shown by (44) so that the mass flux changes slowly. If, on this basis, we set $m \simeq m_{0}$ in the salinity flux equation (19), we find $F_{s}(z) \simeq-m_{0} N^{2} z$. For $w_{b} \ll w$, the relation (20) for the neutral height $L_{n}$ then shows that

$$
L_{n} \simeq \frac{Q_{b} g}{m_{0} N^{2}}=\frac{\sqrt{5}}{2}\left(S^{5 / 4} \Gamma\right)^{-1 / 2} L_{b}
$$

With the same approximations, the momentum equation can be integrated to find

$$
M^{2}(z) \simeq M_{0}^{2}+m_{0}\left(2 Q_{b} g z-m_{0} N^{2} z^{2}\right),
$$

from which we find an estimate $L_{p}$ for the peel height by setting $M=0$ :

$$
L_{p} \simeq(1+\sqrt{1+S}) \frac{Q_{b} g}{m_{0} N^{2}}=\frac{\sqrt{5}}{2}(1+\sqrt{1+S})\left(S^{5 / 4} \Gamma\right)^{-1 / 2} L_{b} .
$$

Large values of $\left(S^{5 / 4} \Gamma\right)^{1 / 2}$ correspond to large $m_{0}^{*}$ and, therefore, to large injection of heavy fluid at the source which decrease both the neutral and the peel heights. Figure 7 , in which the three families of lines correspond to different values of $S^{5 / 4} \Gamma$ and the two curves in each family differ by the value of $S$, refers to this case. The peel height (left panel) mainly depends on $V_{N}$ and $m_{0}^{*}$. The weakness of the dependence on $S$ suggested by the previous derivations becomes particularly evident for small $V_{N}$, in which case the plume behaves like a single homogeneous medium, and agrees with the analysis of the appendix for the single-phase case [see, in particular. Eq. (A16)]. As $V_{N}$ increases, the effect of bubble buoyancy decreases and that of the source momentum becomes comparatively more appreciable.

We now turn to study the interaction of the bubble-induced buoyancy with the source mass and momentum when these effects are dominant in determining the peel height so that $S$ is no longer 
small. We distinguish between strong and weak entrainment cases. In the former, $m \sim 2 \alpha_{e} \sqrt{\pi M_{0}} L$, while in the latter, $m \sim m_{0}$. These two estimates are equal for

$$
L_{\mathrm{sw}}=\frac{m_{0}}{2 \alpha_{e} \sqrt{\pi M_{0}}}=\frac{2}{\sqrt{5 \sqrt{\pi}}} \sqrt{\Gamma S^{3 / 4}} L_{b} .
$$

Upon comparing the length scales to be derived presently with $L_{\mathrm{sw}}$ we can decide between estimates appropriate for the strong and weak entrainment cases.

For both strong and weak entrainment, the buoyancy flux equation (19) gives the estimate $F_{s} \sim-m N^{2} L$, with $L$ a suitable length scale for the peel height, different in the two limits. With the neglect of the bubble buoyancy, which is now a second-order effect in the momentum equation (18), we have $-M_{0} / L \sim F_{s} / w=F_{s} m / M_{0} \sim-m^{2} N^{2} L / M_{0}$, so that

$$
L=\frac{M_{0}}{m N}
$$

If entrainment is strong, from the mass equation (17) we have $m \sim 2 \sqrt{\pi} \alpha_{e} \sqrt{M_{0}} L_{p \text {,se }}$ so that the peel height in the strong-entrainment limit becomes

$$
L_{p, \mathrm{se}}=\left(\frac{M_{0}}{4 \pi \alpha_{e}^{2} N^{2}}\right)^{1 / 4}=S^{1 / 8} L_{b}=L_{m},
$$

which is the same result found earlier in (25) for momentum-dominated single-phase plumes. Entrainment is indeed strong, and this estimate justified, provided that $L_{\mathrm{se}}>L_{\mathrm{sw}}$, i.e.,

$$
\frac{L_{p, \mathrm{se}}}{L_{\mathrm{sw}}}=\frac{\sqrt{5 \sqrt{\pi}}}{2}\left(\Gamma S^{1 / 2}\right)^{-1 / 2}>1 .
$$

By setting $m=m_{0}$ in (54), which would be appropriate for weak entrainment, we find the following estimate for the peel height:

$$
L_{p, \text { we }}=\frac{M_{0}}{m_{0} N}=\frac{\sqrt{5 \sqrt{\pi}}}{2}\left(S^{1 / 2} \Gamma\right)^{-1 / 2} L_{m}=\frac{\sqrt{5 \sqrt{\pi}}}{2}\left(S^{1 / 4} \Gamma\right)^{-1 / 2} L_{b} .
$$

Again, this is justified provided $L_{p \text {,we }}<L_{\text {sw }}$, i.e.,

$$
\frac{L_{p, \mathrm{we}}}{L_{\mathrm{sw}}}=\frac{5 \sqrt{\pi}}{4}\left(\Gamma S^{1 / 2}\right)^{-1}<1 .
$$

Figure 8 shows the dimensionless peel height for strong (left panel) and weak entrainment. In the former case, the peel height is made dimensionless by dividing by $L_{b} S^{1 / 8}$ as suggested by (55), while in the latter case, the quantity plotted is $\sqrt{S^{1 / 4} \Gamma} h_{p} / L_{b}$ as suggested by (57) [see also (A16) in the appendix]. Although the curves in the strong entrainment case get closer as $\sqrt{S^{1 / 2} \Gamma}$ decreases, a convincing proof of their coalescence as $\sqrt{S^{1 / 2} \Gamma}$ decreases implied by (55) would require yet smaller values of the parameter and, while it is consistent with the results shown, it is less than evident. For weak entrainment, (57) suggests that the curves coalesce for increasing $\sqrt{S^{1 / 2} \Gamma}$, which is also confirmed by the numerical results shown. Since, in these conditions, the source momentum is far more important than the bubble buoyancy, $V_{N}$ has only a weak effect on the peel height.

In the conditions to which the previous considerations and Fig. 8 refer, the neutral height is much smaller than the peel height, but we can estimate it starting from (20) and using the previous estimates for $F_{s}$. For strong entrainment, again setting $L_{n} \sim Q_{b} g /\left(m N^{2}\right)$ with $m \sim 2 \sqrt{\pi} \alpha_{e} \sqrt{M_{0}} L_{n}$, we have

$$
L_{n, \text { se }}=\left(\frac{Q_{b}^{2} g^{2}}{4 \pi \alpha_{e}^{2} N^{4} M_{0}}\right)^{1 / 4}=S^{-1 / 8} L_{b}
$$



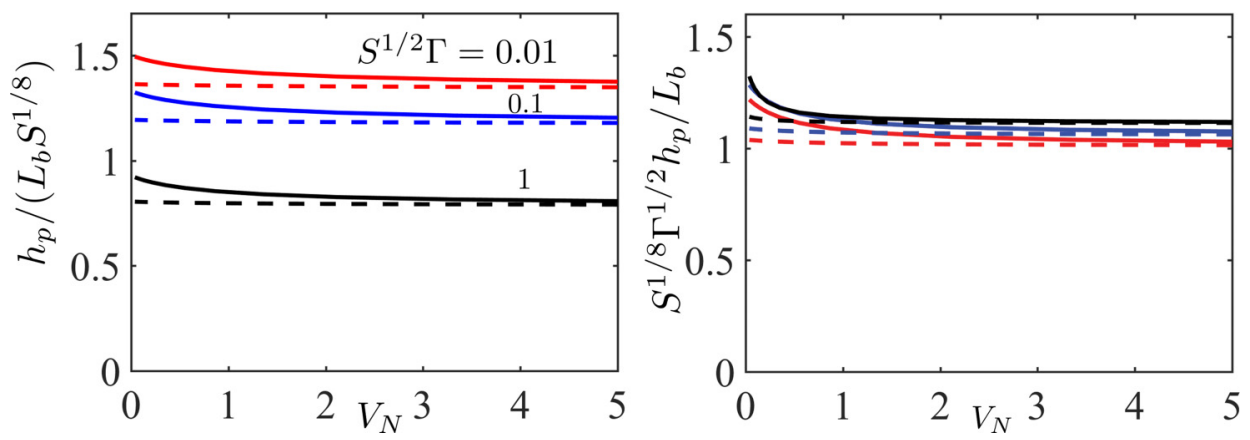

FIG. 8. Left: Dimensionless peel height for strong entrainment. In ascending order, the three pairs of curves correspond to $\Gamma S^{1 / 2}=1,0.1$ and 0.01 . As the value of this parameter decreases, the curves coalesce, validating the scaling argument given in (55). Right: Dimensionless peel height for weak entrainment. In ascending order, the three pairs of curves correspond to $\Gamma S^{1 / 2}=5,10$, and 100. As this parameter increases, the curves collapse as expected from (57). In both figures, the solid lines are for $S=20$ and the dashed lines are for $S=1000$.

a result that is justified provided

$$
\frac{L_{n, \mathrm{se}}}{L_{\mathrm{sw}}}=\frac{\sqrt{5 \sqrt{\pi}}}{2}(\Gamma S)^{-1 / 2}>1 .
$$

When plotted as $h_{n} /\left(S^{-1 / 8} L_{b}\right)=S^{1 / 8} h_{n} / L_{b}$ as suggested by this relation, the neutral height tends to become approximately independent of $S \Gamma$ for small values of this combination. This trend can be discerned in Fig. 9(a) for very small values of $V_{N}$; much smaller values of $S \Gamma$ would be necessary to demonstrate it more fully. The neutral height $L_{n \text {,we }}$ for weak entrainment is the same as (50) found before; this estimate is justified provided that

$$
\frac{L_{n, \mathrm{we}}}{L_{\mathrm{sw}}}=\frac{5 \sqrt{\pi}}{4}(\Gamma S)^{-1}<1 .
$$

The neutral height for this case, normalized as suggested by (50) [see also (A17) in the appendix], is shown in Fig. 9(b). The coalescence of the curves for very small $V_{N}$, expected from (50), is more evident than in Fig. 9(a). Since the neutral height is determined by the competition between the bubble and salinity buoyancy, unlike the peel height, it maintains a strong dependence on $V_{N}$ even
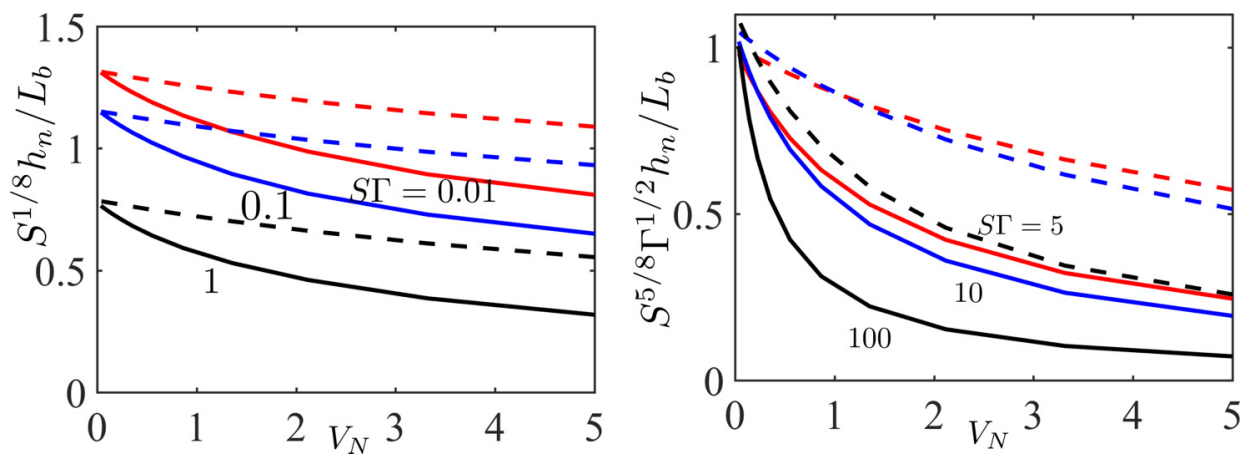

FIG. 9. Left: Dimensionless neutral height for strong entrainment. In ascending order, the three pairs of curves correspond to $\Gamma S=1,0.1$, and 0.01 . The scaling of (59) for small $V_{N}$ is not apparent as it requires yet smaller values of $\Gamma S$. Right: Dimensionless neutral height for weak entrainment. In descending order, the three pairs of curves correspond to $\Gamma S=5,10$, and 100 . The scaling of (50) is evident for very small bubble rise velocity $V_{N}$. In both figures the solid lines are for $S=20$ and the dashed lines for $S=1000$. 
TABLE I. Summary of the scalings for the peel and neutral heights for strong and weak entrainment in the presence of significant momentum injection by the source, with the applicability condition for each result. The parameters $\Gamma$ and $S$ are defined in (38) and (39), respectively. The numerical constant is $C=\sqrt{5 \sqrt{\pi}} / 2 \simeq$ 1.4885 .

\begin{tabular}{|c|c|c|c|c|}
\hline & \multicolumn{2}{|c|}{ Strong entrainment } & \multicolumn{2}{|c|}{ Weak entrainment } \\
\hline & & condition & & condition \\
\hline Peel height & $S^{1 / 8} L_{b}$ & $C\left(\Gamma S^{1 / 2}\right)^{-1 / 2}>1$ & $C\left(S^{1 / 4} \Gamma\right)^{-1 / 2} L_{b}$ & $C^{2}\left(\Gamma S^{1 / 2}\right)^{-1}<1$ \\
\hline Neutral height & $S^{-1 / 8} L_{b}$ & $C(\Gamma S)^{-1 / 2}>1$ & $C\left(S^{5 / 4} \Gamma\right)^{-1 / 2} L_{b}$ & $C^{2}(\Gamma S)^{-1}<1$ \\
\hline
\end{tabular}

when the source momentum is large as demonstrated by (20). Table I summarizes the results of the scaling analysis just presented.

\section{COMPARISON WITH EXPERIMENT}

We now consider how and to what extent the present theory can be compared with experiment and other theoretical analyses. In the presentation of results in the previous section, it was useful to use the parameter $V_{N}$, defined in (37), to nondimensionalize the bubble velocity and the parameter $L_{b}$, defined in (33), to nondimensonalize the neutral and intrusion heights because use of these parameters does not require the explicit adoption of a specific value for the empirical entrainment coefficient $\alpha_{e}$. However, in turning to actual data, it is preferable to follow most authors (see, e.g., Refs. $[15,19,35,39])$ and use a different dimensionless bubble velocity

$$
U_{N}=\frac{w_{b}}{\left(Q_{b} g N\right)^{1 / 4}}=\frac{V_{N}}{\left(4 \pi \alpha_{e}^{2}\right)^{1 / 4}}
$$

and characteristic length

$$
H_{c}=\left(\frac{Q_{b} g}{N^{3}}\right)^{1 / 4}=\left(4 \pi \alpha_{e}^{2}\right)^{1 / 4} L_{b} .
$$

To obtain the results described below, we took $\alpha_{e}=0.117$, which is equivalent to $\alpha_{e}=0.083$ for a Gaussian profile; this is a value widely used in the literature (see, e.g., Refs. [18,41,44]) and close to the average value 0.086 reported in Ref. [35]. With this value of $\alpha_{e}, V_{N} / U_{N} \simeq 0.624$ and $L_{b} / H_{c} \simeq 1.60$. The appearance of the entrainment coefficient $\alpha_{e}$ raised to the power $1 / 2$ in (62) and (63) and in several of the dimensionless parameters introduced in Secs. III and IV indicates that the results are only weakly dependent on this quantity. The only exception is the parameter $\Gamma$, which is inversely proportional to $\alpha_{e}$. We show the sensitivity of our numerical predictions to this quantity later in Table V.

We begin with a comparison with the numerical results of Ref. [35], who carried out LES numerical simulations of bubble plumes in a stratified environment. These are the most suitable results we have found to compare with as, in their Table 1, these authors also report values for the source mass and momentum flow rates $m_{0}, M_{0}$, in addition to the gas flow rate $Q_{b}$ and bubble size. The results of the comparison are shown in Table II. Our predictions are close, although they fall somewhat below the data reported in Ref. [35] for reasons which will be addressed shortly.

Figure 10 shows a collection of experimental results for the intrusion height from various sources rendered dimensionless in terms of the characteristic quantities defined in (62) and (63); the original data, taken from the original papers, are shown in Table III. The dashed line is the theoretical result of Ref. [39] to which we return later. These data exhibit a significant scatter, not only among different investigators but also within the same experimental group. Furthermore, it was noted before in connection with Figs. 1 and 4 that the values of the neutral height corresponding to $S=0$ is the maximum possible. This level, shown by the upper solid line in Fig. 11 (also shown earlier in Fig. 1 
TABLE II. Comparison between the present results for the neutral height $h_{n}$ (last column) and the numerical results for the intrusion height $h_{i}$ reported in Ref. [35]. The values of $S, \Gamma$, and dimensionless bubble rise velocity $V_{N}$ shown are obtained from the information given in the reference.

\begin{tabular}{lcccc}
\hline \hline & & & \multicolumn{2}{c}{$h_{i, n} /\left(Q_{b} g / N^{3}\right)^{1 / 4}$} \\
\cline { 4 - 5 }$U_{N}$ & $\Gamma$ & $S \times 10^{4}$ & {$[35]$} & Present \\
\hline 0.53 & 416 & 1.1 & 2.37 & 2.30 \\
1.06 & 343 & 0.71 & 2.10 & 2.14 \\
2.12 & 267 & 0.53 & 1.95 & 1.89 \\
3.53 & 90 & 0.89 & 1.77 & 1.65 \\
\hline
\end{tabular}

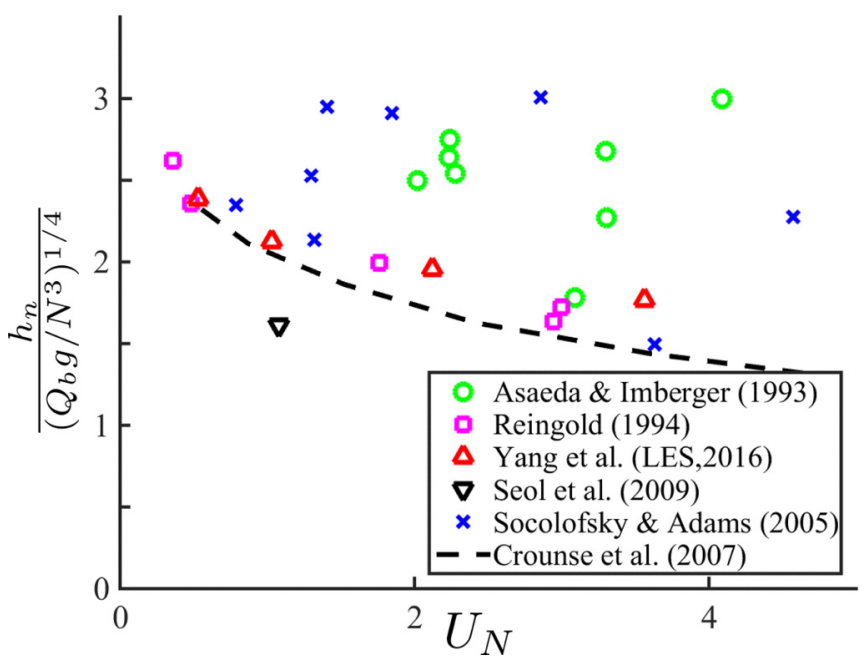

FIG. 10. Normalized intrusion height $h_{i} / H_{c}$ as a function of the dimensionless bubble velocity $U_{N}$ reported by several investigators; the dashed line is the theoretical prediction of Ref. [39].

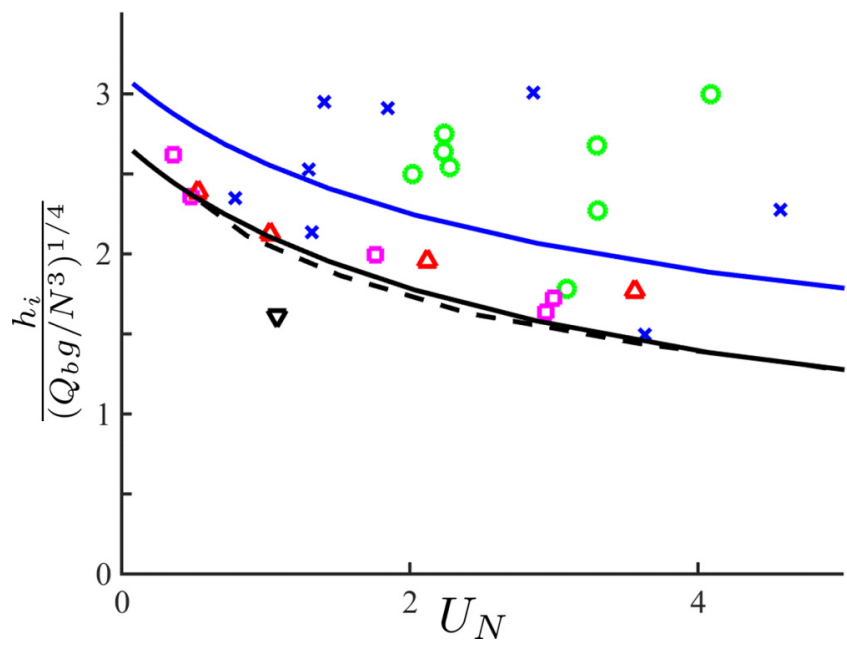

FIG. 11. Normalized intrusion height $h_{i} / H_{c}$ as a function of the dimensionless bubble velocity $U_{N}$. The data points and the dashed line are the same as shown in Fig. 10. The solid lines are the results of the present theory for the neutral height, the lower one for $\Gamma=1$ and $S=0.01$ and the upper one for $S=0$; this latter line is the same as shown with a different normalization in Fig. 1. 
TABLE III. Original data used to generate Fig. 10. The values of $Q_{b} g$ and $w_{b}$ for the data of Ref. [18] have been obtained from the values of $P_{N}$ and $M_{H}$ given in Table 1 of that paper; the value of $D$ shown corresponds to the maximum area reported to be ejecting bubbles in their experiment; $h_{l}$ and $h_{i}$ are the positions of the lower edge of the intrusion layer and the intrusion depth, respectively, reported in Ref. [18]; the other authors only report $h_{i}$. The question mark indicates the tank depth; the depth of the liquid is not reported by these authors.

\begin{tabular}{lcccccccc}
\hline \hline Reference & ID & $\begin{array}{c}N \\
\left(\mathrm{~s}^{-1}\right)\end{array}$ & $\begin{array}{c}10^{6} Q_{b} g \\
\left(\mathrm{~m}^{4} / \mathrm{s}^{3}\right)\end{array}$ & $\begin{array}{c}w_{b} \\
(\mathrm{~m} / \mathrm{s})\end{array}$ & $\begin{array}{c}D \\
(\mathrm{~mm})\end{array}$ & $\begin{array}{c}h_{l} \\
(\mathrm{~m})\end{array}$ & $\begin{array}{c}h_{i} \\
(\mathrm{~m})\end{array}$ & Depth \\
\hline$[18]$ & $\mathrm{C} 1$ & 0.412 & 5.968 & 0.080 & 4.570 & 0.132 & 0.240 & 0.60 \\
& $\mathrm{C} 2$ & 0.274 & 19.151 & 0.158 & 4.570 & 0.312 & 0.468 & 0.60 \\
& $\mathrm{C} 4$ & 0.349 & 4.692 & 0.080 & 4.570 & 0.144 & 0.282 & 0.60 \\
& $\mathrm{~S} 1$ & 0.423 & 4.406 & 0.075 & 4.570 & 0.108 & 0.308 & 0.40 \\
& S2 & 0.349 & 7.017 & 0.090 & 4.570 & 0.176 & 0.288 & 0.36 \\
& S3 & 0.296 & 18.937 & 0.150 & 4.570 & 0.220 & 0.293 & 0.38 \\
& CC & 0.297 & 5.563 & 0.080 & 4.570 & 0.180 & 0.319 & 0.58 \\
& L4 & 0.361 & 74.349 & 0.238 & & 0.302 & 0.454 & 0.56 \\
& L5 & 0.389 & 29.922 & 0.239 & & 0.188 & 0.450 & 0.57 \\
& L015 & 0.310 & 199.000 & 0.070 & & & 0.700 & $2.4(?)$ \\
& T04 & 0.360 & 66.300 & 0.072 & & & 0.390 & $2.4(?)$ \\
& T033 & 0.310 & 26.300 & 0.072 & & & 0.370 & $2.4(?)$ \\
& Air1 & 0.310 & 19.900 & 0.072 & & & 0.440 & $2.4(?)$ \\
& Air2 & 0.310 & 19.900 & 0.072 & & & 0.440 & $2.4(?)$ \\
& Air4 & 0.310 & 19.900 & 0.072 & & & 0.440 & $2.4(?)$ \\
& Air5 & 0.310 & 133.000 & 0.233 & & & 0.740 & $2.4(?)$ \\
& g50a & 0.250 & 66.300 & 0.233 & & & 0.380 & $2.4(?)$ \\
& Air3 & 0.310 & 19.900 & 0.233 & & & 0.340 & $2.4(?)$ \\
\hline \hline & air1 $~ 4 ~$ & 0.480 & 35.000 & 0.190 & 2 & & 0.225 & $0.80(?)$ \\
& & 0.640 & 16.300 & 0.060 & 14 & & 0.143 & $0.80(?)$ \\
& & & & & & & &
\end{tabular}

and reproduced here with the present slightly different normalization), is $h_{n} / H_{c} \simeq 3$ for $U_{N}=0$ and decreases with increasing $U_{N}$. It will be observed in Fig. 10 that several of the data points violate this upper bound, and not only for $U_{N}$ close to 0 but also for such large values of $U_{N}$ that $h_{n} / H_{c}$ should be less than half the maximum. As experimental errors of this magnitude are unlikely, there must be another explanation for these apparent inconsistencies.

All the data shown in Fig. 10 refer to bubble plumes generated by injecting only gas, but no liquid, at the source. Taken literally, this would imply that the inlet quantities $m_{0}$ and $M_{0}$ of our model should both vanish and, with them, also $S$ and $\Gamma$. This conclusion, however, would be incorrect as no integral model can be expected to hold in the flow-establishment zone near the source where the self-similarity assumption does not apply.

One way to deal with this problem, proposed in Ref. [39], consists in calculating effective values for $m_{0}$ and $M_{0}$ at the source from

$$
m_{0}=\frac{6}{5}\left(\frac{9 \pi^{2}}{10} \alpha_{e}^{4} Q_{b} g z_{0}^{5}\right)^{1 / 3}, \quad M_{0}=\left(\frac{81 \pi}{100} \alpha_{e}^{2} Q_{b}^{2} g^{2} z_{0}^{4}\right)^{1 / 3},
$$

by taking $z_{0}=10 D$, with $D$ as the source diameter. These are the relations for a fully developed single-phase plume at a distance $z_{0}$ from a point source (see, e.g., Ref. [46]). If these relations are converted to the parameters $\Gamma$ and $S$ of the present theory, we find $\Gamma=1$ and

$$
S=270\left(\frac{10 \pi^{2}}{3} \frac{\alpha_{e}^{4} D^{8}}{Q_{b}^{2} g^{2}}\right)^{1 / 3}
$$




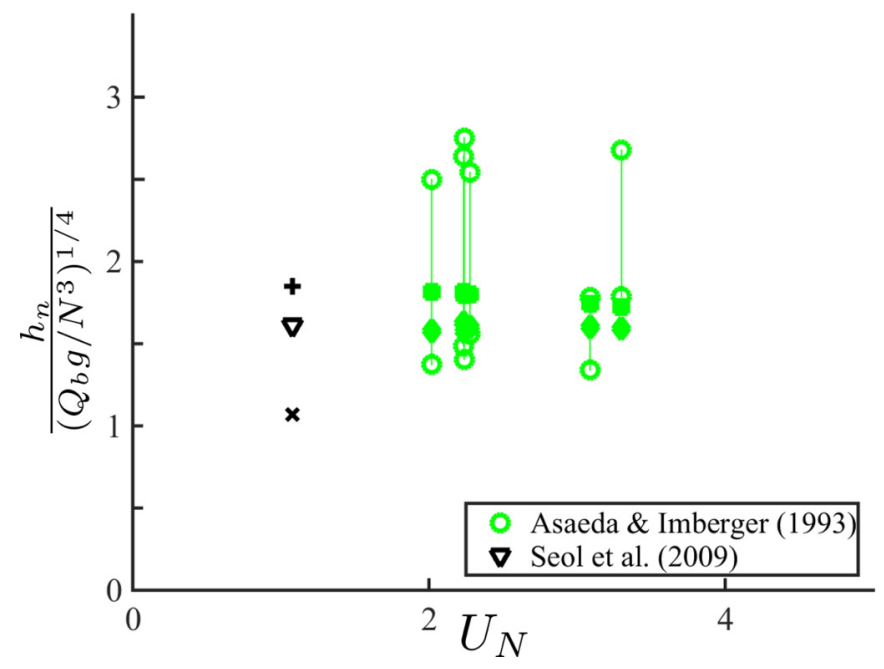

FIG. 12. The vertical lines connect the data points of Ref. [18], marking the lower edge of the intrusion layer and the intrusion height. The filled squares and diamonds are the corresponding predictions of the neutral height given by the present theory with and without the flow-establishment zone correction. The open triangle is the single data point reported in Ref. [13], and the symbols above and below it are the present results with and without the flow-establishment zone correction.

The normalized intrusion height $h_{i} / H_{c}$ reported in Fig. 3 of Ref. [39] is reproduced as the dashed line in Figs. 10 and 11 as a function of the dimensionless bubble velocity $U_{N}$. It is not straightforward to compare this result with the predictions of the present theory as there is some uncertainty as to the actual values of $D$ and $Q_{b}$ used in Ref. [39] but, upon taking $S=0.01$, we find the good match shown by the solid line in Fig. 11. If we use the data shown in Fig. 6 of Ref. [39], namely $U_{N}=$ $3, w_{b}=0.12 \mathrm{~m} / \mathrm{s}$, and $N=0.08 \mathrm{~s}^{-1}$, we find $Q_{b}=3.27 \times 10^{-6} \mathrm{~m}^{3} / \mathrm{s}=196 \mathrm{~mL} / \mathrm{s}$, which, for $S$ $=0.01$, corresponds to $D \simeq 21 \mathrm{~mm}$, which appears reasonable. The results of Refs. [35] (upward triangles) and [51] (squares) shown in Fig. 11 are between the lines for $S=10^{-2}$ and that for $S=0$ and can easily be fitted by the present model. However, there is no way to fit most of the other data. There are two observations to make in this connection.

In the first place, a proper comparison of data with theory should allow for the presence of a flow-establishment zone traversing which the plume attains its ultimate self-similar structure as the liquid is gradually accelerated by the relatively weak drag exerted by the bubbles. A meaningful comparison with the present theory, which relies on the assumption of self-similarity, is only possible starting from the top of this zone. In this perspective, the proper values of $m_{0}$ and $M_{0}$ to be used in the theory are the values of these quantities at the top of this flow-establishment zone. This correction is not necessary if there is a significant liquid injection at the source, as in the calculation of Ref. [35], but it is a central problem when the liquid mass and momentum fluxes at the source vanish.

An estimate of the height of the flow-establishment zone can be attempted following Ref. [12]. This author gives two estimates for the height $z_{E}$ of the flow-establishment zone but only one of them applies in the vast majority of cases. According to this estimate, $z_{E} \simeq 5 D$, which is also consistent with later work (see, e.g., Ref. [37]). From this estimate, Ref. [12] deduces a value of $M_{0}$ given by

$$
M_{0} \simeq \frac{2 Q_{b}}{w_{b}} g z_{E}
$$

Upon using the top-hat relations (16), we then find

$$
m_{0}=b_{0} \sqrt{M_{0}},
$$


TABLE IV. Values of the dimensionless parameters $\Gamma$ and $S$ according to (68) for the Milgram procedure and to (65) for the procedure of Crounse et al. (in which case $\Gamma=1$ ); $U_{N}$ is defined in (62). The column labeled "Exp" shows the normalized measured values; the quantities $h_{i}$ and $h_{l}$ are explained in the caption of the previous table; $h_{n}$ is the neutral height of the present theory. The last two pairs of columns show the dimensionless intrusion heights calculated according to the two procedures, uncorrected and corrected by the addition of the height of the flow-establishment zone (f.e.z.) estimated to be $5 D$.

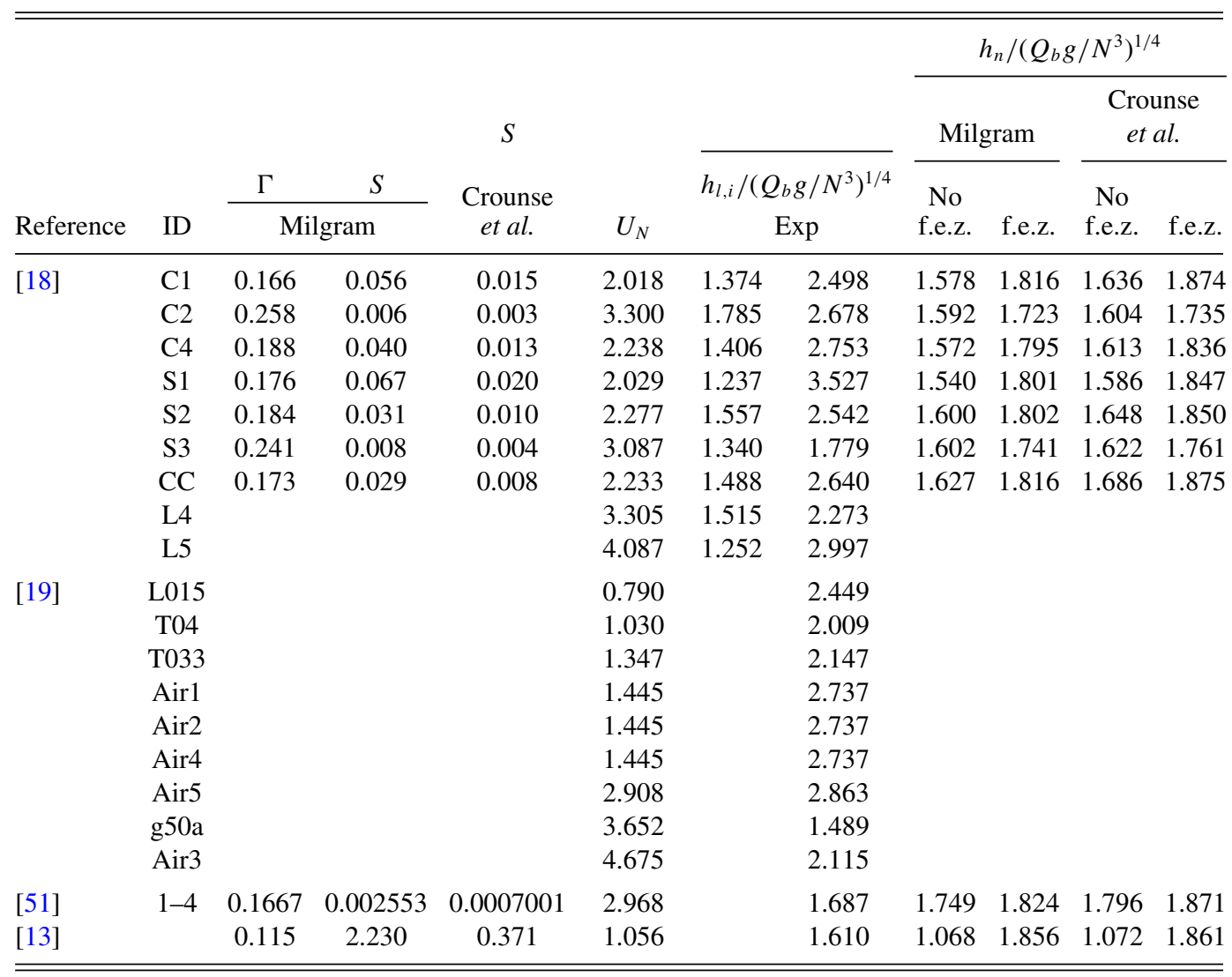

in which, from the spreading rate of a single-phase jet [46], $b_{0} \simeq \frac{1}{2} D+2 \alpha_{e} z_{E}$. With these results, we can calculate the dimensionless parameters $S$ and $\Gamma$ :

$$
S=100 \frac{D^{2} N^{2}}{w_{b}^{2}}, \quad \Gamma=\frac{\left(1+20 \alpha_{e}\right)^{2}}{60 \alpha_{e}} \sqrt{\frac{w_{b}^{3} D}{Q_{b} g}},
$$

from which we also find

$$
\Gamma^{1 / 2} S^{-1 / 8}=\frac{\left(1+20 \alpha_{e}\right) w_{b}}{8 \sqrt{5}\left(4 \pi \alpha_{e}^{2} Q_{b} N\right)^{1 / 4}}=\frac{1+20 \alpha_{e}}{8 \sqrt{5}} V_{N} .
$$

The second, and possibly more important, point is that, as noted in Sec. II, the neutral height predicted by the model should actually be considered as an estimate of the lower edge of the intrusion layer. Reference [18] includes experimental results not only for the intrusion height but also for the lower edge of the intrusion layer. These data are shown in Table III and in Fig. 12, where they are connected by a vertical line to the respective reported intrusion height. In this figure, we show our predictions for the neutral height without correction for the zone of flow establishment by a filled diamond and with the correction by a filled square. We see that there is a good overall match between 
TABLE V. Effect of the entrainment coefficient on the neutral height predicted by the present model for the data shown in Fig. 12. The first three columns repeat information given in Table IV and identify the data point, the normalized measured intrusion layer lower edge, and intrusion depth. The fourth and fifth columns are the predictions of the present model for values of the entrainment coefficient $20 \%$ smaller and larger than those used to generate Fig. 12. The last two columns are the corresponding values of the parameter $\Gamma$.

\begin{tabular}{|c|c|c|c|c|c|c|}
\hline \multirow{2}{*}{$\begin{array}{l}\text { Data } \\
\text { point }\end{array}$} & \multicolumn{2}{|c|}{ Experimental } & \multicolumn{2}{|c|}{ Model $h_{n} /\left(Q_{b} g / N^{3}\right)^{1 / 4}$} & \multicolumn{2}{|c|}{$\Gamma$} \\
\hline & $h_{i} /\left(Q_{b} g / N^{3}\right)^{1 / 4}$ & $h_{l} /\left(Q_{b} g / N^{3}\right)^{1 / 4}$ & $\alpha_{e}=0.0664$ & $\alpha_{e}=0.0996$ & $\alpha_{e}=0.0664$ & $\alpha_{e}=0.0996$ \\
\hline $\mathrm{C} 1$ & 1.374 & 2.498 & 1.833 & 1.393 & 0.154 & 0.180 \\
\hline $\mathrm{C} 2$ & 1.785 & 2.678 & 1.852 & 1.403 & 0.238 & 0.279 \\
\hline $\mathrm{C} 4$ & 1.406 & 2.753 & 1.825 & 1.387 & 0.174 & 0.204 \\
\hline S1 & 1.237 & 3.527 & 1.789 & 1.359 & 0.162 & 0.190 \\
\hline $\mathrm{S} 2$ & 1.557 & 2.542 & 1.858 & 1.414 & 0.170 & 0.199 \\
\hline S3 & 1.340 & 1.779 & 1.864 & 1.414 & 0.222 & 0.260 \\
\hline $\mathrm{CC}$ & 1.488 & 2.640 & 1.889 & 1.438 & 0.159 & 0.187 \\
\hline Seol & & 1.610 & 1.244 & 0.941 & 0.106 & 0.124 \\
\hline
\end{tabular}

predictions and observations. The open triangle is the single data point reported in Ref. [13], and the symbols above and below it are the present results with and without the establishment zone correction.

The predictions of the current model are affected by the specific value used for the entrainment coefficient $\alpha_{e}$, which is not firmly established. To give an idea of the sensitivity of the predictions to this parameter, we show in Table $\mathrm{V}$ the present predictions for the neutral height for values of $\alpha_{e}$ decreased and increased by $20 \%$ for the same cases shown in Fig. 12. The first three columns identify the data point and repeat the measured values of the lower edge of the intrusion layer, $h_{l}$, and of the intrusion layer, $h_{i}$, from Table IV. The last two columns show the values of $\Gamma$ corresponding to $\alpha_{e}$. The model predictions, shown in the fourth and fifth columns, have been calculated without including the height of the flow establishment zone.

As expected, a decrease or an increase of $\alpha_{e}$ result in a higher and lower level of the neutral zone, respectively. The higher levels shown in the table are all below the reported intrusion height except for the data point S3 which, however, seems anomalous upon comparison with the other measured values. The lower levels corresponding to the larger $\alpha_{e}$ are mostly above, or just slightly below, the reported lower edge of the intrusion layer. It may be concluded that the present model is consistent with the available data, even though the accuracy of the data does not permit a stringent test given the uncertainty of the value of $\alpha_{e}$.

Reference [18] is the only data set reporting information not only on the intrusion height, but also on the position of the lower edge of the intrusion layer. This key distinction appears to be essential not only for the data of Ref. [18], but also for most of those of Ref. [19] which fall well above predictions as shown by the crosses in Fig. 11 and which seem to violate established upper bounds as noted before. Other data are closer to the model predictions. One may conjecture that, in these latter cases, the thickness of the intrusion layer was relatively small. Unfortunately, in the absence of more complete information on the experimental observations, it is not possible to reach a firmer conclusion.

All the data considered so far are from laboratory experiments. Some field data have become available in connection with the Deepwater Horizon accident. Unfortunately the actual conditions prevailing in the course of the accident differed from those envisaged in our model. In particular, the measured dependence of the salinity stratification with depth was closer to quadratic than linear [52], but Ref. [54] argues for the applicability of an effective Brunt-Väisäla frequency approximately given by $N=1.5 \times 10^{-3} \mathrm{~s}^{-1}$. The plume discharged oil in addition to gas but, with $Q_{b} g=0.80 \mathrm{~m}^{4} / \mathrm{s}^{3}$, the latter provided more than $80 \%$ of the total buoyancy flux. With $D=0.5 \mathrm{~m}, w_{b}=0.21 \mathrm{~m} / \mathrm{s}$ 
[52], and $\alpha_{e}=0.117$ as before, we find $S=0.0013, \Gamma=0.115$, and $U_{N}=1.10$. The predicted neutral height from our model is $h_{n}=291 \mathrm{~m}$, in good agreement with the trap height reported in Ref. [52], which is in the range from 307 to $366 \mathrm{~m}$. It must be observed, however, that the result is sensitively dependent on the value of $N$ used, ranging between 767 and $186 \mathrm{~m}$ as $N$ is increased from $0.4 \times 10^{-3}$ to $2.7 \times 10^{-3} \mathrm{~s}^{-1}$.

\section{SUMMARY AND CONCLUSIONS}

In this paper, we have developed a horizontally integrated model for bubble plumes in stratified environments. By a suitable rescaling of the dependent and independent variables, the equations of the model take the same form for a top-hat and a Gaussian distribution of the flow fields over the plume cross section. We have provided a scaling analysis of the dependence of the neutral and peel heights on the main dimensionless parameters arising in the theory. These parameters are connected to the conditions prevailing at the plume source and therefore, unlike earlier models, our study includes the effects of inlet conditions.

Another difference with much earlier work (see, e.g., Refs. $[15,19,28]$ ), is that we were careful to make a distinction between the height at which the plume momentum attains its maximum value, which we term the neutral height, and the intrusion height reported in many experiments. We have argued that the neutral height is always lower than the intrusion height of which it represents, at best, the lower edge, and cannot be taken to represent the measured intrusion height. This distinction proves critical when it is attempted to compare the results of our model, as well as those of earlier investigators, with experimental data, which would otherwise be found to fall inexplicably much above the theoretical predictions violating basic physical constraints in the majority cases.

\section{ACKNOWLEDGMENTS}

This research was made possible by a grant from The Gulf of Mexico Research Initiative (USA), DROPPS-II CONSORTIUM. Data are publicly available through the Gulf of Mexico Research Initiative Information and Data Cooperative (GRIIDC) at https://data.gulfresearchinitiative.org doi:10.7266/N7H9939V.

\section{APPENDIX}

The purpose of this appendix is to provide some additional details on several aspects of the methods and results summarized in the main body of this paper.

\section{Derivation of (12)}

Upon integrating the liquid momentum equation over the plume cross section, we have

$$
\frac{d}{d z} \int_{A}\left(1-\alpha_{b}\right) \rho u_{z}^{2} d A=\int_{A}\left[(1-\alpha)\left(\rho_{a}-\rho\right) g+n f_{z, \text { bub }}\right] d A-\tau_{t} \mathcal{P}
$$

in which $f_{z \text {,bub }}$ is the force exerted by a bubble on the liquid, $n$ is the bubble number density, $\tau_{t}$ is the turbulent stress at the edge of the plume, and $\mathcal{P}$ is the perimeter of the plume. After a similar integration, the momentum equation for the bubble phase is

$$
\frac{d}{d z} \int_{A} \alpha_{b} \rho_{\text {bubble }} u_{\text {bubble }}^{2} d A=\int_{A}\left[\alpha_{b}\left(\rho-\rho_{\text {bubble }}\right) g-n f_{z, \text { bub }}\right] d A .
$$

Upon adding these two equations and neglecting the density of the bubbles, the result may be written as

$$
\frac{d}{d z} \int_{A}\left(1-\alpha_{b}\right) \rho u_{z}^{2} d A=\int_{A}\left[\alpha_{b}\left(2 \rho-\rho_{a}\right) g+\left(\rho_{a}-\rho\right) g\right] d A-\tau_{t} \mathcal{P}
$$


or approximately, upon setting $\rho \simeq \rho_{w}$,

$$
\frac{d}{d z} \int_{A}\left(1-\alpha_{b}\right) u_{z}^{2} d A=\int_{A}\left[\alpha_{b} \frac{2 \rho-\rho_{a}}{\rho_{w}} g+\frac{\rho_{a}-\rho}{\rho_{w}} g\right] d A-\tau_{t} \mathcal{P}
$$

which is (12) after dropping the small effect due to turbulent drag at the edge of the plume.

\section{Solution for a single-phase plume in uniform ambient}

The solution for a single-phase buoyant plume in a stratified ambient liquid has been worked out in Ref. [29] in the case in which the plume issues from a pointlike source or, equivalently, the plume has a pointlike virtual origin. Here we extend this solution to the case of a source of finite diameter. We assume a top-hat profile which permits us to write $B_{s}=F_{s} / w$ as directly follows from (16). In dimensionless form, the governing equations for the single-phase case are then (44) and (46), while the momentum equation (45) is modified to

$$
\frac{d M^{*}}{d z^{*}}=\frac{m^{*} F_{s}^{*}}{M^{*}}
$$

The inlet conditions are $m^{*}=m_{0}^{*}, M^{*}=M_{0}^{*}$, and $F_{s}^{*}=F_{s 0}^{*}=1$. As noted in Sec. V, the solution that follows can also be applied to the case of (49) in which the bubbles rise so slowly that the bubbly mixture behaves like a light homogeneous liquid. As shown by (49), for this application it is sufficient to replace $F_{s}^{*}$ in (A5) by $F_{s}^{*}+1$, in which $F_{s}^{*}(0)=0$. Upon multiplying (45) by $M^{*}$, (46) by $F_{s}^{*}$ (or, in the two-phase case, by $F_{s}^{*}+1$ ), adding, and integrating, we find

$$
\left(M^{*}\right)^{2}+\left(F_{s}^{*}\right)^{2}=\left[M^{*}(0)\right]^{2}+\left[F_{s}^{*}(0)\right]^{2}=S+1,
$$

with $S$ defined in (26). This relation shows that $M^{*}$ reaches a maximum value $\sqrt{S+1}$ when $F_{s}^{*}=0$, i.e., at the neutral height $h_{n}^{*}$. As the plume continues its rise by inertia, $F_{s}^{*}$ turns negative and $M^{*}$ starts decreasing until it vanishes at the peel height $h_{p}^{*}$. By manipulating (44) and (45), we have

$$
2 m^{*} d m^{*}=\frac{\sqrt{M^{*}}}{\sqrt{S+1-\left(M^{*}\right)^{2}}} d\left(M^{*}\right)^{2} .
$$

Upon integration, from the inlet to the neutral height we find

$$
(S+1)^{-3 / 4}\left[m^{* 2}\left(h_{n}\right)-m_{0}^{* 2}\right]=\beta\left[\frac{5}{4}, \frac{1}{2} ; \frac{\left(M^{*}\right)^{2}}{S+1}\right]-\beta\left(\frac{5}{4}, \frac{1}{2} ; \frac{S}{S+1}\right),
$$

where $\beta$ is the incomplete $\beta$ function. A further integration from the neutral height to the peel height gives

$$
(S+1)^{-3 / 4}\left[m^{* 2}\left(h_{p}^{*}\right)-m^{* 2}\left(h_{n}^{*}\right)\right]=2 \beta\left(\frac{5}{4}, \frac{1}{2}\right)-\beta\left[\frac{5}{4}, \frac{1}{2} ; \frac{\left(M^{*}\right)^{2}}{S+1}\right]-\beta\left(\frac{5}{4}, \frac{1}{2} ; \frac{S}{S+1}\right) .
$$


With $m^{*}$ known as a function of $M^{*}$, we can find expressions for the neutral and peel heights from (44). The results are

$$
\begin{gathered}
\frac{h_{p}}{L_{b}}=\frac{1}{2}(1-\sigma)^{-1 / 8}\left(I_{1}+I_{2}\right), \\
\frac{h_{n}}{L_{b}}=\frac{1}{2}(1-\sigma)^{-1 / 8} I_{1},
\end{gathered}
$$

where

$$
\begin{gathered}
I_{1}(\sigma, \phi)=\int_{\sigma}^{1} \frac{d t}{\sqrt{(1-t)\left[\phi^{2}+\beta\left(\frac{5}{4}, \frac{1}{2}, t\right)-\beta\left(\frac{5}{4}, \frac{1}{2}, \sigma\right)\right]}}, \\
I_{2}(\sigma, \phi)=\int_{0}^{1} \frac{d t}{\sqrt{(1-t)\left[\phi^{2}+2 \beta\left(\frac{5}{4}, \frac{1}{2}\right)-\beta\left(\frac{5}{4}, \frac{1}{2}, t\right)-\beta\left(\frac{5}{4}, \frac{1}{2}, \sigma\right)\right]}}
\end{gathered}
$$

with $\sigma=S /(S+1)$ and

$$
\phi=\frac{m_{0}^{*}}{(S+1)^{3 / 8}}=\frac{2}{\sqrt{5}} \Gamma^{1 / 2} \frac{S^{5 / 8}}{(S+1)^{3 / 8}}=\left(\frac{M_{0}^{2} N^{2}}{M_{0}^{2} N^{2}+F_{0}^{2}}\right)^{3 / 8} \frac{m_{0} N^{1 / 2}}{\left(4 \pi \alpha_{e}^{2} M_{0}^{3}\right)^{1 / 4}} .
$$

As the parameter $t$ ranges from 0 to 1 , the incomplete $\beta$ function $\beta\left(\frac{5}{4}, \frac{1}{2}, t\right)$ ranges from 0 to $\beta\left(\frac{5}{4}, \frac{1}{2}\right) \simeq 1.748$.

The integrals can be simplified when $\phi$ is large to find

$$
I_{1} \simeq \frac{2}{\phi \sqrt{S+1}}, \quad I_{2} \simeq \frac{2}{\phi}
$$

For small $S$ (but $\Gamma$ large enough that $\phi$ is large) (A10) and (A11) become

$$
\begin{gathered}
\frac{h_{p}}{L_{b}} \simeq \frac{2}{\phi}=\sqrt{\frac{5}{\Gamma S^{5 / 4}}} \\
\frac{h_{n}}{L_{b}} \simeq \frac{1}{\phi}=\frac{1}{2} \sqrt{\frac{5}{\Gamma S^{5 / 4}}}=\frac{1}{2} \frac{h_{p}}{L_{b}},
\end{gathered}
$$

in agreement with (52)and (50). For large $S$, on the other hand, (A10) and (A11) give

$$
\begin{gathered}
\frac{h_{p}}{L_{b}} \simeq \frac{S^{1 / 8}}{\phi}=\frac{\sqrt{5}}{2 \sqrt{\Gamma S^{1 / 4}}} \\
\frac{h_{n}}{L_{b}} \simeq \frac{1}{\phi S^{3 / 8}}=\frac{\sqrt{5}}{2 \sqrt{\Gamma S^{5 / 4}}}
\end{gathered}
$$

in agreement with (57) and (50).

For a point source $\Gamma=0$, for $S=0, \sigma=0$, and $\phi=0$, the numerical evaluation of the integrals gives $I_{1} \simeq 3.91$ and $I_{2} \simeq 1.24$. With these values, we find the results for the neutral height quoted at the end of Sec. III. For large $S, I_{1} \rightarrow 0$ while $I_{2} \simeq 2.86$. 


\section{Gaussian profiles}

Assume

$$
\begin{gathered}
w(r, z)=w(r=0, z) e^{-r^{2} / b^{2}}, \\
\frac{\rho(r, z)-\rho_{a}(z)}{\rho_{w}} g=\frac{\rho(r=0, z)-\rho_{a}}{\rho_{w}} g e^{-r^{2} /\left(\lambda_{s} b\right)^{2}}, \\
\alpha_{b}(r, z)=\alpha_{b}(z) e^{-r^{2} /\left(\lambda_{b} b\right)^{2}} .
\end{gathered}
$$

Then we have

$$
\begin{gathered}
m=\pi w b^{2}, \\
M=\frac{1}{2} \pi w^{2} b^{2}, \\
B_{f}=\pi\left(\lambda_{s} b\right)^{2} \frac{\rho-\rho_{a}}{\rho_{w}} g, \\
F_{s}=\pi \frac{\lambda_{s}^{2}}{1+\lambda_{s}^{2}} w b^{2} \frac{\rho-\rho_{a}}{\rho_{w}} g, \\
B_{b}=\pi \alpha_{b} g\left(\lambda_{b} b\right)^{2}, \\
F_{b}=B_{b}\left(\frac{w}{1+\lambda_{b}^{2}}+w_{b}\right)=Q_{b} g,
\end{gathered}
$$

with $w, \rho$, and $\alpha_{b}$ evaluated on the axis of the plume. Similar expressions have also been obtained, e.g., Ref. [4,15] for bubble plumes, Ref. [29] for single-phase plumes, and Ref. [53] for a descending particle plume.

With the Gaussian profiles, the dimensional governing equations become

$$
\begin{gathered}
\frac{d m}{d z}=2 \alpha_{e} \sqrt{2 \pi M}, \\
\frac{d M}{d z}=B_{b}+B_{s}=\frac{Q_{b} g}{w /\left(1+\lambda_{b}^{2}\right)+w_{b}}+\left(1+\lambda_{s}^{2}\right) \frac{F_{s}}{w}, \\
\frac{d F_{s}}{d z}=-m N^{2} .
\end{gathered}
$$

Since $F_{s}<0$, increasing $\lambda_{s}$ decreases the peel height. An intuitive view at the dimensional momentum equation would show that increasing $\lambda_{s}$ will decrease the peel level, whereas increasing $\lambda_{b}$ will increase the peel level.

We can recast the dimensionless version of these equation in a form identical to the one used before with the following definitions:

$$
\begin{gathered}
m^{*}=\frac{m}{\pi \sqrt{2\left(1+\lambda_{s}^{2}\right)} N \tilde{L}_{b}\left(2 \alpha_{e} \tilde{L}_{b}\right)^{2}}, \quad M^{*}=\frac{M}{\pi\left(1+\lambda_{s}^{2}\right) N^{2} \tilde{L}_{b}^{2}\left(2 \alpha_{e} \tilde{L}_{b}\right)^{2}}, \\
B_{b}^{*}=\frac{F_{b}}{\pi \sqrt{2\left(1+\lambda_{s}^{2}\right)} N^{2} \tilde{L}_{b}\left(2 \alpha_{e} \tilde{L}_{b}\right)^{2}}, \quad F_{s}^{*}=\frac{F_{s}}{\pi \sqrt{2\left(1+\lambda_{s}^{2}\right)} N^{3} \tilde{L}_{b}^{2}\left(2 \alpha_{e} \tilde{L}_{b}\right)^{2}}, \\
V_{N}=\frac{1+\lambda_{b}^{2}}{\sqrt{2\left(1+\lambda_{s}^{2}\right)}} \frac{w_{b}}{N \tilde{L}_{b}},
\end{gathered}
$$


in which

$$
\tilde{L}_{b}=\left(\frac{1+\lambda_{b}^{2}}{\left[2\left(1+\lambda_{s}^{2}\right)\right]^{3 / 2}}\right)^{1 / 4} L_{b}, \quad z^{*}=\frac{z}{\tilde{L}_{b}} .
$$

Upon redefining the parameters $S$ and $\Gamma$ according to

$$
\begin{aligned}
& S=\frac{8\left(1+\lambda_{s}^{2}\right)}{\left(1+\lambda_{b}^{2}\right)^{2}}\left(\frac{M_{0} N}{Q_{b} g}\right)^{2}, \\
& \Gamma=\frac{5\left(1+\lambda_{b}^{2}\right)}{2^{11 / 2} \sqrt{\pi}} \frac{Q_{b} g m_{0}^{2}}{\alpha_{e} M_{0}^{5 / 2}},
\end{aligned}
$$

the previous expressions (47) for $m_{0}^{*}$ and $M_{0}^{*}$ are recovered. The $\Gamma$ defined in Ref. [38] for single-phase Gaussian plumes is twice as large as that defined here with $\lambda_{b}$ replaced by $\lambda_{s}$. We adopt this slightly different definition so that the form of the dimensionless equations is the same as for the top-hat profile.

[1] L. Boegman and S. Sleep, Feasibility of bubble plume destratification of Central Lake Erie, J. Hydraul. Eng. ASCE 138, 985 (2012).

[2] M. A. Imteaz and T. Asaeda, Artificial mixing of lake water by bubble plume and effects of bubbling operations on algal bloom, Water Res. 34, 1919 (2000).

[3] G. B. Sahoo and D. Luketina, Response of a tropical reservoir to bubbler destratification, J. Environ. Eng. Sci. ASCE 132, 736 (2006).

[4] A. Wüest, N. H. Brooks, and D. M. Imboden, Bubble plume modeling for lake restoration, Water Res. Res. 28, 3235 (1992).

[5] T. Baumberger, M. D. Lilley, J. A. Resing, J. E. Lupton, E. T. Baker, D. A. Butterfield, E. J. Olson, and G. L. Fruh-Green, Understanding a submarine eruption through time series hydrothermal plume sampling of dissolved and particulate constituents: West Mata, 2008-2012, Geochem. Geophy. Geosy. 15, 4631 (2014).

[6] C. Zhang, S. Eckert, and G. Gerbeth, The flow structure of a bubble-driven liquid-metal jet in a horizontal magnetic field, J. Fluid Mech. 575, 57 (2007).

[7] M. K. McNutt, R. Camilli, T. J. Crone, G. D. Guthrie, P. A. Hsieh, T. B. Ryerson, O. Savas, and F. Shaffer, Review of flow rate estimates of the Deepwater Horizon oil spill, Proc. Natl. Acad. Sci. USA 109, 20260 (2012).

[8] C. M. Reddy, J. S. Arey, J. S. Seewald, S. P. Sylva, K. L. Lemkau, R. K. Nelson, C. A. Carmichael, C. P. McIntyre, J. Fenwick, G. T. Ventura, B. A. S. van Mooy, and R. Camilli, Composition and fate of gas and oil released to the water column during the Deepwater Horizon oil spill, Proc. Natl. Acad. Sci. USA 109, 20229 (2012).

[9] T. B. Ryerson, R. Camilli, J. D. Kessler, E. B. Kujawinski, C. M. Reddy, D. L. Valentine, E. Atlas, D. R. Blake, J. de Gouw, S. Meinardi, et al., Chemical data quantify Deepwater Horizon hydrocarbon flow rate and environmental distribution, Proc. Natl. Acad. Sci. USA 109, 20246 (2012).

[10] F. A. Bombardelli, G. C. Buscaglia, C. R. Rehmann, L. E. Rincón, and M. H. García, Modeling and scaling of aeration bubble plumes: A two-phase flow analysis, J. Hydraul. Res. ASCE 45, 617 (2007).

[11] A. M. Leitch and W. D. Baines, Liquid volume flux in a weak bubble plume, J. Fluid Mech. 205, 77 (1989).

[12] J. H. Milgram, Mean flow in round bubble plumes, J. Fluid Mech. 133, 345 (1983). 
[13] D. G. Seol, D. B. Bryant, and S. A. Socolofsky, Measurement of behavioral properties of entrained ambient water in a stratified bubble plume, J. Hydraul. Eng. ASCE 135, 983 (2009).

[14] T. J. McDougall, Bubble plumes in stratified environments, J. Fluid Mech. 85, 655 (1978).

[15] S. A. Socolofsky, T. Bhaumik, and D. G. Seol, Double-plume integral models for near-field mixing in multiphase plumes, J. Hydraul. Eng. ASCE 134, 772 (2008).

[16] L. J. Bloomfield and R. C. Kerr, A theoretical model of a turbulent fountain, J. Fluid Mech. 424, 197 (2000).

[17] G. R. Hunt and H. C. Burridge, Fountains in industry and nature, Annu. Rev. Fluid Mech. 47, 195 (2015).

[18] T. Asaeda and J. Imberger, Structure of bubble plumes in linearly stratified environments, J. Fluid Mech. 249, 35 (1993).

[19] S. A. Socolofsky and E. E. Adams, Role of slip velocity in the behavior of stratified multiphase plumes, J. Hydraul. Eng. ASCE 131, 273 (2005).

[20] Y. Murai and Y. Matsumoto, Numerical study of the three-dimensional structure of a bubble plume, ASME J. Fluids Eng. 122, 754 (2000).

[21] I. E. L. Neto, D. Z. Zhu, and N. Rajaratnam, Effect of tank size and geometry on the flow induced by circular bubble plumes and water jets, J. Hydraul. Eng. ASCE 134, 833 (2008).

[22] J. Rensen and V. Roig, Experimental study of the unsteady structure of a confined bubble plume, Int. J. Multiphase Flow 27, 1431 (2001).

[23] M. Simiano, D. Lakehal, M. Lance, and G. Yadigaroglu, Turbulent transport mechanisms in oscillating bubble plumes, J. Fluid Mech. 633, 191 (2009).

[24] M. Simiano, R. Zboray, F. De Cachard, D. Lakehal, and G. Yadigaroglu, Comprehensive experimental investigation of the hydrodynamics of large-scale, 3D, oscillating bubble plumes, Int. J. Multiphase Flow 32, 1160 (2006).

[25] C. J. Lemckert and J. Imberger, Energetic bubble plumes in arbitrary stratification, J. Hydraul. Eng. ASCE 119, 680 (1993).

[26] U. C. Bandara and P. D. Yapa, Bubble sizes, breakup, and coalescence in deepwater gas/oil plumes, J. Hydraul. Eng. ASCE 137, 729 (2011).

[27] L. Zhao, M. C. Boufadel, K. Lee, T. King, N. Loney, and X. Geng, Evolution of bubble size distribution from gas blowout in shallow water, J. Geophys. Res. Oceans 121, 1573 (2016).

[28] L. Zheng, P. D. Yapa, and F. Chen, A model for simulating deepwater oil and gas blowouts, part I: Theory and model formulation, J. Hydraul. Res. ASCE 41, 339 (2003).

[29] B. R. Morton, Forced plumes, J. Fluid Mech. 5, 151 (1959).

[30] B. R. Morton, G. Taylor, and J. S. Turner, Turbulent gravitational convection from maintained and instantaneous sources, Proc. Roy. Soc. London, Ser. A 234, 1 (1956).

[31] C. H. B. Priestley and F. K. Ball, Continuous convection from an isolated source of heat, Quart. J. Roy. Met. Soc. 81, 144 (1955).

[32] G. C. Buscaglia, F. A. Bombardelli, and M. H. García, Numerical modeling of large-scale bubble plumes accounting for mass transfer effects, Int. J. Multiphase Flow 28, 1763 (2002).

[33] B. Fraga and T. Stoesser, Influence of bubble size, diffuser width, and flow rate on the integral behavior of bubble plumes, J. Geophys. Res.-Oceans 121, 3887 (2016).

[34] B. Fraga, T. Stoesser, C. C. K. Lai, and S. A. Socolofsky, An LES-based Eulerian-Lagrangian approach to predict the dynamics of bubble plumes, Ocean Model. 97, 27 (2016).

[35] D. Yang, B. Chen, S. A. Socolofsky, M. Chamecki, and C. Meneveau, Large-eddy simulation and parameterization of buoyant plume dynamics in stratified flow, J. Fluid Mech. 794, 798 (2016).

[36] A. F. Tomas, A. Fabregat, A. C. Poje, T. M. Ozgokmen, and W. K. Dewar, Dynamics of multiphase turbulent plumes with hybrid buoyancy sources in stratified environments, Phys. Fluids 28, 095109 (2016).

[37] J. H. W. Lee and V. Chu, Turbulent Jets and Plumes-A Lagrangian Approach (Kluwer Academics Publishers, Dordrecht, The Netherlands, 2003).

[38] B. R. Morton and J. Middleton, Scale diagrams for forced plumes, J. Fluid Mech. 58, 165 (1973).

[39] B. C. Crounse, E. J. Wannamaker, and E. E. Adams, Integral model of a multiphase plume in quiescent stratification, J. Hydraul. Eng. ASCE 133, 70 (2007). 
[40] C. R. Liro, E. E. Adams, and H. J. Herzog, Modeling the release of $\mathrm{CO}_{2}$ in the deep ocean, Energ. Convers. Manage. 33, 667 (1992).

[41] G. R. Hunt and N. B. Kaye, Lazy plumes, J. Fluid Mech. 533, 329 (2005).

[42] L. Landau and E. M. Lifshitz, Fluid Mechanics, 2nd ed. (Pergamon Press, Oxford, UK, 1989).

[43] S. B. Pope, Turbulent Flows (Cambridge University Press, Cambridge, UK, 2000).

[44] J. S. Turner, Turbulent entrainment: The development of the entrainment assumption and its application to geophysical flows, J. Fluid Mech. 173, 431 (1986).

[45] G. Carazzo, E. Kamiski, and S. Tait, The route to self-similarity in turbulent jets and plumes, J. Fluid Mech. 547, 137 (2006).

[46] M. van Reeuwijk, P. Salizzoni, G. R. Hunt, and J. Craske, Turbulent transport and entrainment in jets and plumes: A DNS study, Phys. Rev. Fluids 1, 074301 (2016).

[47] G. Riboux, F. Risso, and D. Legendre, Experimental characterization of the agitation generated by bubbles rising at high Reynolds number, J. Fluid Mech. 643, 509 (2010).

[48] M. M. Scase, C. P. Caulfield, and S. B. Dalziel, Boussinesq plumes and jets with decreasing source strengths in stratified environments, J. Fluid Mech. 563, 463 (2006).

[49] J. S. Turner, Buoyancy Effects in Fluids (Cambridge University Press, Cambridge, UK, 1979).

[50] E. J. List, Turbulent jets and plumes, Annu. Rev. Fluid Mech. 14, 189 (1982).

[51] L. S. Reingold, An experimental comparison of bubble and sediment plumes in stratified environments. $\mathrm{Ph} . D$. thesis, Massachusetts Institute of Technology, Cambridge, MA, 1994 (unpublished).

[52] W. Lehr, S. Bristol, and A. Possolo, Oil budget calculator-Deepwater Horizon, technical documentation: A report to the National Incident Command. Tech. rep., Coastal Response Research Center, Durham, NH (The Federal Interagency Solutions Group, Oil Budget Calculator Science and Engineering Team, 2010).

[53] A. C. H. Lai, S. N. Chan, A. W. K. Law, and E. E. Adams, Spreading hypothesis of a particle plume, J. Hydraul. Eng. ASCE 142, 04016065 (2016).

[54] S. A. Socolofsky, E. E. Adams, and C. R. Sherwood, Formation dynamics of subsurface hydrocarbon intrusions following the Deepwater Horizon blowout, Geophys. Res. Lett. 38, L09602 (2011). 